$$
\begin{gathered}
\text { UNIVERSIDADE DE BRASÍLIA } \\
\text { FACULDADE DE EDUCAÇÃO FÍSICA } \\
\text { PROGRAMA DE PÓS-GRADUAÇÃO STRICTO-SENSU EM } \\
\text { EDUCAÇÃO FÍSICA }
\end{gathered}
$$

\title{
AVALIAÇÃO DA EXCITABILIDADE NEUROMUSCULAR EM PACIENTES CRITICAMENTE ENFERMOS
}

PAULO EUGÊNIO SILVA

BRASÍLIA 
AVALIAÇÃO DA EXCITABILIDADE NEUROMUSCULAR EM PACIENTES CRITICAMENTE ENFERMOS

PAULO EUGÊNIO SILVA

Dissertação apresentada à Faculdade de Educação Física da Universidade de Brasília, como requisito parcial para a obtenção do grau de Mestre em Educação Física.

ORIENTADOR: PROF. DR. JOÃO LUIZ QUAGLIOTTI DURIGAN 


\title{
AVALIAÇÃO DA EXCITABILIDADE NEUROMUSCULAR EM PACIENTES CRITICAMENTE ENFERMOS
}

\begin{abstract}
Dissertação aprovada como requisito parcial para obtenção do título de Mestre em Educação Física pelo Programa de Pós Graduação da Faculdade de Educação Física da Universidade de Brasília.
\end{abstract}

Banca Examinadora:

Prof. Dr. João Luiz Quagliotti Durigan

(Orientador - FEF/UnB)

\begin{tabular}{|c|}
\hline $\begin{array}{l}\text { Prof. Dr. Alexandre Simões Dias } \\
\text { (Examinador Externo - UFRGS) }\end{array}$ \\
\hline $\begin{array}{c}\text { Prof. Dr. Rinaldo Mezzarane } \\
\text { (Examinador Interno - FEF/UnB) }\end{array}$ \\
\hline $\begin{array}{c}\text { Prof. Dr. Rodrigo Carregaro } \\
\text { (Examinador Suplente - UnB/FCE) }\end{array}$ \\
\hline
\end{tabular}

Brasília - DF, de de 2015 


\section{DEDICATÓRIA}

Esta dissertação é dedicada a toda minha família. À minha esposa, Alessandra Vasconcelos de Queiroz por ter acreditado no meu sonho e pela coragem em me acompanhar até Brasília. Aos meus filhos, Paulo Vasconcelos Silva e Louise Vasconcelos Silva por suportarem a minha grande ausência em todo este período. À minha mãe, Maria José Silva pela imensa dedicação que sempre teve às minhas causas, principalmente às mais difíceis.

Contudo, esta dissertação é dedicada em especial ao meu pai, Paulo Humberto Oliveira Silva. Além de ter me ensinado desde criança, não só com palavras, mas com atitudes, o valor do conhecimento e dos estudos, me deu todo o apoio moral e financeiro para completar mais esta etapa. 


\section{AGRADECIMENTOS}

São muitas as pessoas que merecem meus agradecimentos neste momento. Gostaria de agradecer ao meu orientador, o Prof. João Durigan por ter acreditado no meu projeto, nada teria acontecido se em setembro de 2013 quando tivemos o primeiro contato por skype, ele tivesse recusado a minha ideia. Ele conseguiu extrair o máximo de mim, me colocou no limite diversas vezes e eu sobrevivi. Depois de tudo, me sinto muito mais preparado.

Meus agradecimentos vão para os parceiros do Hospital de Base do Distrito Federal os quais foram fundamentais para que tudo fluísse bem em todo período de coleta. Ao colega Hedian Gomes pela grande ajuda na fase inicial do estudo.

Gostaria de agradecer a todos da Universidade de Brasília que contribuíram com este projeto. Vale ressaltar a significativa colaboração do Prof. Gerson Cipriano Jr.

Eu me senti extremamente grato com a atitude dos professores da banca examinadora que prontamente aceitaram o convite para colaborar com esta dissertação: muito obrigado Prof. Alexandre Simões Dias, Prof. Rinaldo Mezzarane e Prof. Rodrigo Carregaro.

Não posso deixar de agradecer a todos os colegas do Grupo de Pesquisa Plasticidade Músculo Tendínea do qual faço parte. Sempre muito queridos e incentivadores. Preciso ressaltar o meu agradecimento para Karina Livino e Amaro Eduardo os quais estão diretamente ligados a esta linha de pesquisa e vêm realizando um excelente trabalho.

Por fim, meu profundo agradecimento à Fundação de Apoio a Pesquisa do Distrito Federal (FAP-DF) que financiou esta pesquisa e à Coordenação de Aperfeiçoamento de Pessoal de Nível Superior (CAPES) a qual financiou a minha bolsa de mestrado. 
SUMÁRIO

Página

LISTA DE FIGURAS ................................................................... vii

LISTA DE SIGLAS …........................................................................ viii

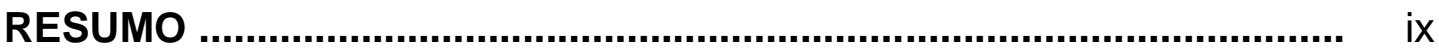

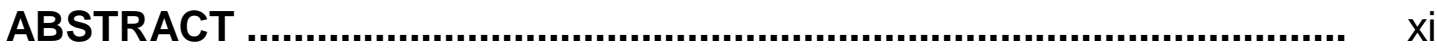

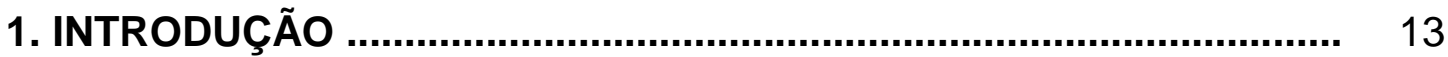

2. REVISÃO DE LITERATURA …...................................................... 15

2.1 Traumatismo cranioencefálico .................................................. 15

2.2 Fraqueza adquirida na unidade de terapia intensiva ..................... 17

2.3 Avaliação eletrofisiológia do doente crítico .................................. 19

2.4 Avaliação da estrutura muscular por ultrassonografia ................. 24

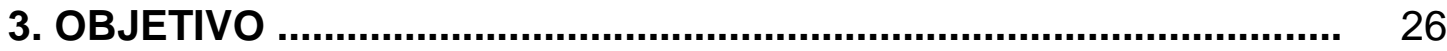

4. MATERIAIS E MÉTODOS ............................................................. 27

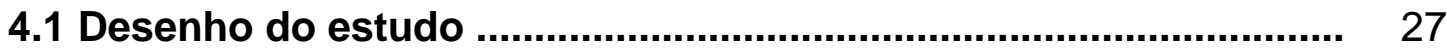

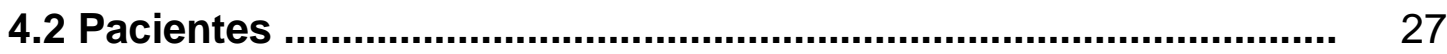

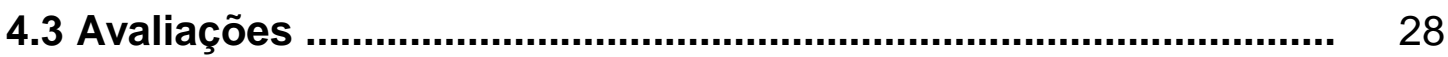

4.3.1 Teste de Eletrodiagnóstico de estímulo ................................... 28

4.3.2 Ultrassonografia ........................................................................ 29

4.4 Análise estatística ....................................................................... 31

5. RESULTADOS .......................................................................... 32

5.1 Características de base e fluxo do estudo .................................... 32

5.2 Dados eletrofisiológicos ........................................................... 33

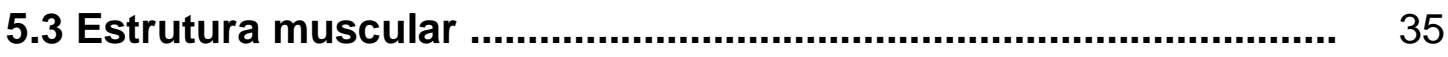

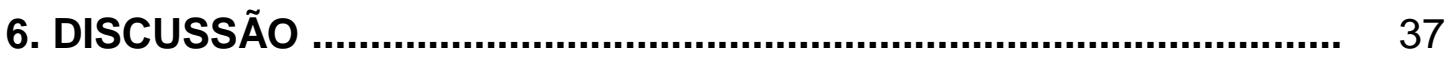

7. CONCLUSÃO …..................................................................... 43

REFERÊNCIAS …..................................................................... 44

ANEXO I - Aprovação do Comitê de Ética em Pesquisa com Seres Humanos 


\section{LISTA DE FIGURAS E TABELAS}

Página

Figura 1 - Desenho do estudo e descrição das avaliações realizadas ... 27

Figura 2 - Locais de mensuração da espessura muscular .................... $\quad 30$

Figura 3 - Diagrama de Fluxo...................................................... 33

Figura 4 - Avaliação da excitabilidade neuromuscular ............... 34

Figura 5 - Avaliação ultrassonográfica da espessura e ecogenicidade muscular ................................................................................ $\quad 36$

Tabela 1 - Características de base ...................................................... 32 


\section{LISTA DE SIGLAS}

ANOVA - Análise da variância

APACHE II - Acute Physiology and Chronic Health disease Classification

System II

BB - Bíceps braquial

DP - Desvio padrão

ECN - Estudos de condução nervosa

FA-UTI- Fraqueza adquirida na unidade de terapia intensiva

IIQ - intervalo interquartil

PAMC - Potencial de ação muscular composto

PANS - Potencial de ação do nervo sensorial

RF - Reto femoral

SIRS - Síndrome da resposta inflamatória sistêmica

TA - Tibial anterior

TCE - Traumatismo cranioencefálico

UTI - Unidade de terapia intensiva

VM - Ventilação mecânica 


\section{RESUMO}

Introdução: $O$ traumatismo cranioencefálico (TCE) é um importante problema mundial de saúde pública. $\mathrm{O}$ avanço no tratamento dos pacientes com TCE tem aumentado a taxa de sobrevivência e assim, o número de indivíduos com sequelas funcionais. Ainda na unidade de terapia intensiva (UTI), estes pacientes desenvolvem significativa atrofia muscular. Não está claro se as alterações musculares nestes pacientes estão associadas, além do desuso, às disfunções eletrofisiológicas. A determinação dos limiares de excitabilidade como reobase, cronaxia e acomodação é fundamental podendo auxiliar no tratamento e assim melhorar os resultados. Desta forma, o comportamento eletrofisiológico neuromuscular de pacientes com TCE restritos ao leito precisa ser melhor documentado. Método: Foi realizado um estudo prospectivo observacional para avaliar o comportamento da excitabilidade neuromuscular e estrutura muscular (espessura e ecogenicidade) em pacientes com TCE submetidos à ventilação mecânica. Os pacientes foram avaliados nas primeiras 24 horas de VM e nos dias 3,7 e 14. A excitabilidade neuromuscular foi determinada pela mensuração da reobase, cronaxia, acomodação, além do índice de acomodação. $O$ índice de acomodação é calculado pela relação: acomodação/reobase. A presença de alterações na excitabilidade neuromuscular foi considerada na presença de cronaxia $>1000 \mu$ s e índice de acomodação $<2$. A estrutura muscular foi avaliada por ultrassonografia modo B. Tibial anterior (TA), reto femoral (RF) e bíceps braquial (BB) foram os músculos analisados. Os pacientes foram acompanhados desde o primeiro dia de internação na unidade de terapia intensiva (UTI) até o décimo quarto dia. Resultados: quarenta e nove pacientes foram admitidos e vinte e sete perdas foram contabilizadas no decorrer dos quatorze dias de seguimento (treze óbitos e quatorze altas). Ao final do estudo, vinte e dois pacientes foram analisados. A normalidade dos dados foi testada com o teste de Shapiro Wilk e as variáveis paramétricas foram descritas em média e desvio padrão ( \pm DP). Variáveis não paramétricas foram apresentadas em mediana e IIQ. As diferenças nas cronaxias em cada músculo ao longo dos dias foram medidas pelo teste de 
Friedman ajustado para comparações múltiplas pelo teste de Dunn. Para avaliar as variáveis categóricas (presença ou não de disfunção na excitabilidade neuromuscular, determinado pela presença de cronaxia > $1000 \mu$ s e índice de acomodação < 2), foi utilizado o teste McNemar. Diferenças na espessura muscular e na ecogenicidade em cada musculo ao longo dos dias foram medidas pelo teste de analise de variância (ANOVA) de medidas repetidas ajustada para comparações múltiplas pelo teste post hoc de Tukey. A reobase mediana e intervalo interquartil ao longo dos dias foi de 6 (4-8) mA para TA, 16 (12-24) mA para RF e 5 (3-8) mA para o BB. Diferenças estatisticamente significativas na cronaxia foram detectadas apenas no TA quando se comparou a mediana do dia 1, 300 (200-700) $\mu \mathrm{s}$ vs. dia 14, 500 (250-2000) $\mu \mathrm{s}, \mathrm{p}=0,04$. Não foi observada diferença no RF

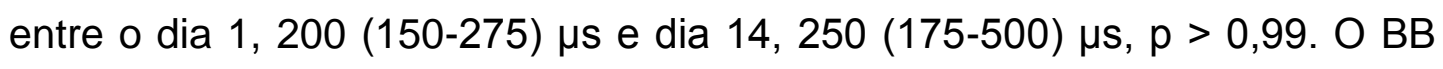
apresentou resultados semelhantes a este ultimo músculo, 150 (70-200) $\mu \mathrm{s}$ e 150 (100-400) $\mathrm{\mu s}$, para o dia 1 e o dia 14, respectivamente, $p>0,99$. A acomodação mediana ao longo dos dias foi de 6 (1-11) mA para TA, 21 (834) $\mathrm{mA}$ para $\mathrm{RF}$ e 9 (2-16) $\mathrm{mA}$ para o BB. $O$ acompanhamento da excitabilidade neuromuscular do dia 1 ao dia 14 mostrou um aumento na taxa absoluta de disfunção da excitabilidade para todos os músculos: $44 \%$ no TA; $29 \%$ no RF e $9 \%$ no BB. No entanto, este aumento só foi estatisticamente significativo no TA, $p=0,01$. Todos os músculos apresentaram diminuição estatisticamente significativa na espessura quando comparados o dia 1 com o dia $14(p<0,05)$. Entretanto, o TA foi o único músculo a apresentar aumento estatisticamente significativo na ecogenicidade entre o primeiro e décimo quarto dia $(p=0.01)$. Conclusão: Pacientes com TCE ventilados mecanicamente não só apresentam alterações na estrutura muscular ao longo da estadia na UTI, mas também, desenvolvem alterações na excitabilidade neuromuscular.

Palavras-chave: atrofia muscular, cronaxia, desuso muscular, estimulação elétrica neuromuscular, fisioterapia, músculo esquelético, reabilitação, reobase, terapia intensiva. 


\section{ABSTRACT}

Introduction: Traumatic brain injury (TBI) is an important global public health problem. The improvement in the management of TBI patients has increased the survival rate and thus the number of individuals with functional dysfunction. Already in the intensive care unit (ICU), these patients develop significant muscle atrophy. It is unclear whether the muscular changes in these patients are associated only to disuse or, beyond it, to electrophysiological disorders. The determination of excitability thresholds as rheobase, chronaxie, and accommodation is the key to improve results. Thus, neuromuscular electrophysiological behavior of TBI patients confined to bed to be better documented. Methods: It was carried out a prospective observational study to investigate the behavior of neuromuscular excitability and muscle structure (thickness and echogenicity) in TBI patients undergoing mechanical ventilation. Patients were evaluated in the first $24 \mathrm{~h}$, and at day 3 , 7 and 14. Neuromuscular excitability was determined by measuring rheobase, chronaxie, and accommodation, besides the accommodation index. The accommodation index is calculated by the relationship: accommodation/rheobase. The determination of neuromuscular excitability dysfunction was considered when chronaxie $>1000 \mu$ s and accommodation index $<2$. The muscle structure was assessed by ultrasound $B$ mode. Tibialis anterior (TA), rectus femoris (RF), and biceps brachialis (BB) muscles were analyzed. Patients were followed from the first day of admission in the intensive care unit (ICU) until the fourteenth day. Results: Forty-nine patients were admitted and twenty-seven losses were recorded in the course of fourteen days of follow-up (thirteen deaths and fourteen discharges). At the end of the study, twenty-two patients were analyzed. Normality of the data was tested with the Shapiro Wilk test and parametric variables were described as the mean plus or minus the standard deviation ( $\pm S D$ ). Nonparametric variables were presented in median and interquartile ranges (IQR). The chronaxie differences of every muscle over the 14 days were evaluated with the Friedman test and adjusted for multiple comparisons with Dunn's test. To evaluate categorical variables (presence or non-presence of 
neuromuscular excitability dysfunction), determined by chronaxie $>1000 \mu \mathrm{s}$ plus accommodation index $<2$, the McNemar test was used. Muscle thickness and echo intensity differences of every muscle over time were measured with repeated-measures ANOVA adjusted for multiple comparisons by Tukey post hoc test. The median and interquartile range rheobase over the days was 6 (4-8) mA for TA, 16 (12-24) mA for RF and 5 (3-8) $\mathrm{mA}$ for BB. Statistically significant differences in chronaxie were detected only in the TA when comparing the median day 1, 300 (200-700) $\mu \mathrm{s}$ vs. day 14,500 (250-2000) $\mu \mathrm{s}, \mathrm{p}=0.04$. No difference was observed in RF between day 1, 200 (150-275) $\mu$ s and day 14, 250 (175-500) $\mu \mathrm{s}, \mathrm{p}>0.99$. BB showed similar results as the last muscle, 150 (70-200) and $150 \mu$ s (100400) $\mu \mathrm{s}$, for day 1 and day 14 , respectively, $p>0.99$. The median accommodation over the days was 6 (1-11) mA for TA, 21 (8-34) mA for RF and $9(2-16) \mathrm{mA}$ to $\mathrm{BB}$. The monitoring of neuromuscular excitability from day 1 to day 14 showed an increasing in absolute rate of neuromuscular excitability dysfunction for all muscles: $44 \%$ in the TA; $29 \%$ in the RF and $9 \%$ in the BB. Although, this increase was statistically significant only at TA $p=$ 0.01 . All muscles showed a statistically significant decrease in thickness when compared with day 1 and day $14(p<0.05)$. However, the TA muscle was the only one to show a statistically significant increase in echogenicity between the first and fourteenth day $(p=0.01)$. Conclusion: Patients with TBI mechanically ventilated not only showed changes in muscle structure along the stay in the ICU, but also developed neuromuscular excitability dysfunction.

Keywords: Accommodation Index; chronaxie; Disuse muscle atrophy; neuromuscular electrical stimulation; Intensive care; Intensive care unitacquired weakness; Physical therapy. 


\section{INTRODUÇÃO}

O traumatismo cranioencefálico (TCE) é um importante problema de saúde pública mundial. É uma das principais causas de incapacidade, morbidade e mortalidade em todas as faixas etárias (1, 2). A melhora no manejo dos pacientes com TCE tem levado ao aumento na taxa de sobrevivência e assim, no número de pessoas com sequelas funcionais (3). $O$ repouso prolongado no leito, no qual os pacientes com TCE são submetidos, é uma variável independente para o desenvolvimento da fraqueza adquirida na unidade (FA-UTI) (4), bem como a perda de massa muscular. Pacientes que evoluem com FA-UTI e perda de massa muscular apresentam piores prognósticos funcionais (5). A FA-UTI é eletrofisiologicamente caracterizada pela redução nas amplitudes do potencial de ação muscular composto (PAMC) e/ou do potencial de ação do nervo sensorial (PANS), além do aumento na latência deste primeiro potencial. (6).

O modo tradicional para aquisição do (PAMC) ocorre por meio da eletromiografia com eletrodo de agulha que é um exame invasivo e demorado, além de difícil realização na UTI. No entanto, uma alternativa para detectar disfunção na excitabilidade neuromuscular é a determinação da reobase, cronaxia e acomodação às quais são adquiridas por meio do teste de eletrodiagnóstico de estímulo, (7-9). Este é um exame não invasivo, com baixo custo, validado para diagnosticar alterações da excitabilidade neuromuscular com sensibilidade variando de 88 a $100 \%$ quando comparado a um método com maior acurácia, a eletromiografia com eletrodo de agulha (7). O teste é conduzido com geradores universais de pulso e se baseia no princípio diagnóstico das diferentes respostas evocadas pelo nervo e pelo musculo com parâmetros de estimulação predeterminados $(7,10)$. O eletrodiagnóstico de estímulo, além de ferramenta diagnóstica, proporciona a aquisição de informações fundamentais para a realização da estimulação elétrica neuromuscular (EENM). A EENM vem sendo considerada a principal ferramenta para interpor os efeitos deletérios do repouso prolongado no leito (11). Estratégias para facilitar a recuperação, minimizar os resultados adversos e promover a saúde geral nos pacientes 
com TCE, são realizadas ainda no período de internação hospitalar $(5,12)$. A eficácia deste tratamento foi demonstrada por uma recente revisão sistemática (11). Nesta revisão, foram analisados oito ensaios clínicos onde foi concluído que a EENM é eficaz para prevenir a fraqueza muscular em pacientes criticamente enfermos. No entanto, os resultados individuais de cada estudo apresentaram grande variabilidade. Dois ensaios não mostraram nenhum impacto da EENM sobre a diminuição da perda de massa e no aumento da força muscular $(13,14)$. A heterogeneidade das características de corrente utilizadas nos protocolos de EENM nestes oito ensaios, dificultaram a interpretação dos resultados e podem estar relacionados com a falta de padronização de protocolos de EENM nesses pacientes (11).

Atualmente, alguns estudos vêm ressaltando a importância de se determinar os protocolos de EENM com as melhores características de corrente para um tratamento mais eficaz $(11,15)$. Entre as principais características recomendadas para melhorar a eficácia da EENM para 0 aumento da força muscular estão: a utilização das maiores intensidades possíveis para evocar o maior pico de torque, o ajuste da frequência em torno de $100 \mathrm{~Hz}$, o tempo de contração mais longo do que 2s, localização do ponto motor e o uso da largura de pulso acima do limiar motor (15). No entanto, até o presente momento, nenhum estudo conduzido na UTI seguiu estas recomendações. É fundamental entender o comportamento da excitabilidade neuromuscular para executar protocolos de EENM mais eficazes, uma vez que o ponto motor e o limiar motor devem ser determinados. Assim, o uso do teste de eletrodiagnóstico de estímulo pode ajudar na aplicação de protocolos de EENM na tentativa de determinar estratégias de estimulação mais racionais $(9,15,16)$.

Baseado nos aspectos já citados, a hipótese desse estudo foi que pacientes criticamente enfermos com TCE além de desenvolverem atrofia muscular, podem cursar com disfunção na excitabilidade neuromuscular. Isso pode diminuir a eficácia dos protocolos de EENM convencionais, bem como os programas de reabilitação precoce que visam melhorar a força e diminuir a perda de massa muscular. 


\section{REVISÃO DE LITERATURA}

\subsection{Traumatismo cranioencefálico}

O traumatismo crânio encefálico (TCE) é considerado um problema de saúde pública mundial. A cada ano ocorre pelo menos 10 milhões de casos graves resultando em mortes e/ou hospitalização (17). A previsão é que o TCE se torne a terceira maior causa de morte e incapacidade em todo o mundo no ano de 2020 (18). O TCE é definido como qualquer lesão decorrente de um trauma externo, que tenha como consequência alterações cerebrais momentâneas ou permanentes, de natureza cognitiva ou funcional (19). Estima-se que 57 milhões de pessoas em todo o mundo tenham sido hospitalizadas por TCE. A proporção de pacientes vivendo com deficiências causadas por este insulto ainda não é bem estabelecida (17). Estima-se que nos Estados Unidos a prevalência seja de 5 milhões de pessoas, isto é, 1$2 \%$ da população têm alguma deficiência causada pelo TCE, enquanto na Europa este numero pode chegar a 7,7 milhões de indivíduos com sequelas (20).

A incidência do TCE em países desenvolvidos apresenta-se em torno de 200 por 100.000 habitantes por ano. Esta estimativa inclui, tipicamente, apenas pacientes com TCE internados em hospitais. Isso resulta numa subnotificação de casos mais leves (21). Nos Estados Unidos, em média, 1,4 milhões de casos ocorrem a cada ano, incluindo 1,1 milhões de atendimentos em serviços de emergência, 235.000 hospitalizações e 50.000 mortes. Na Europa a taxa de incidência é de 235 casos por 100.000 habitantes por ano e uma taxa de mortalidade média de 15 por 100.000 por ano (22). No Brasil, o TCE representa a terceira causa de morte configurando-se como inquestionável desafio aos gestores de políticas públicas, principalmente porque o TCE atinge preponderantemente a camada jovem e produtiva da sociedade (23). De acordo com o DATASUS (www.datasus.gov.br), em 2011, foram realizadas 547.468 internações devido ao TCE resultando em 12.800 óbitos, assim uma taxa de mortalidade de 2,34\% ao ano. Em geral, os homens são cerca de duas vezes mais propensos que as mulheres a sofrerem TCE $(17,23)$. Este padrão é 
explicado principalmente como resultado da violência interpessoal e colisões de veículos automotivos durante a adolescência e início da vida adulta (21). Crianças de 0 a 4 anos, adolescentes e adultos jovens entre 15 e 19 anos, bem como idosos acima de 75 anos, são mais propensos ao TCE (17).As principais causas do TCE são os acidentes automobilísticos, as quedas e as causas violentas. Os acidentes automobilísticos correspondem a (50\%) dos casos. Neste grupo, a principal faixa etária é de adolescentes e adultos jovens. Dos 15 aos 24 anos, os acidentes de trânsito são responsáveis por mais mortes que todas as outras causas juntas. As quedas são responsáveis por $30 \%$ dos casos com um grande número de idosos. As causas violentas de TCE correspondem a $20 \%$ dos casos. Normalmente, estão associadas aos ferimentos por projétil de arma de fogo e as armas brancas. Outras causas que também contribuem para o TCE são os acidentes ocorridos durante os esportes e o lazer (23).

Estima-se que o TCE seja a principal causa de morte dentre todas as causas traumáticas (24). Entretanto, pesquisas demonstram que a taxa de mortalidade no TCE vem diminuindo $(9,10)$. A melhora no manejo destes pacientes vem sendo demonstrada pela significativa redução na mortalidade nos últimos 30 anos, passando de 50 para 25\% mesmo quando ajustado para a severidade do trauma, idade e parâmetros prognósticos na admissão (25). Assim, o numero de pacientes com sequelas funcionais vem aumentando a cada ano e se tornando um problema de saúde publica. $O$ TCE pode promover a longo prazo sequelas físicas, cognitivas, comportamentais e emocionais. Mesmo o TCE leve, incluindo a concussão, pode causar problemas físicos e cognitivos que afetam a capacidade do indivíduo realizar atividades diárias e retornar ao trabalho $(27,28)$. Como resultado, o TCE é uma das lesões mais incapacitantes. A perda na produtividade chega a ser 14 vezes maior nos pacientes com sequelas de TCE do que nos pacientes com lesão na medula espinal (17).

De acordo com um importante guideline, é decisivo reconhecer que os danos causados pelo TCE não ocorrem somente no momento do impacto, mas sim nas horas e dias subsequentes ao trauma (29). Isso inclui todo o período de internação na UTI. Em pacientes com TCE grave, a incidência de 
infecção é aumentada com a utilização da ventilação mecânica e da monitorização hemodinâmica invasiva. Foi demonstrado que mais de $70 \%$ dos pacientes podem desenvolver pneumonia associada à ventilação e a incidência de infecção foi $27 \%$ maior nos pacientes com monitorização invasiva aumentando a necessidade do uso de antibióticos (29). A atuação dos cuidados de fisioterapia dentro da equipe multidisciplinar tem um papel fundamental para minimizar estas complicações. O processo de reabilitação após o TCE é geralmente longo (23). Na fase aguda da reabilitação, ainda na UTI, o objetivo é garantir o manejo respiratório adequado e evitar as complicações neuromusculares (5,30). A abordagem precoce pode promover menor perda de massa muscular e assim diminuir as sequelas decorrentes da imobilização no leito (11).

O TCE impõe um enorme impacto à sociedade. Os custos incluindo despesas médicas e perda de produtividade, podem chegar a bilhões de dólares em todo o mundo. Isso sem contar com o impacto indireto sobre amigos, familiares e cuidadores $(17,31)$. Considerando os dados expostos, 0 TCE deve ser visto como uma doença da sociedade moderna, presente em todo o território brasileiro e em todas as idades, acometendo homens e mulheres. É necessário que sejam criados mecanismos para minimizar o impacto de suas consequências.

\subsection{Fraqueza adquirida na unidade de terapia intensiva}

A FA-UTI resulta de uma mistura complexa entre patologias musculares e dos nervos periféricos (32). A FA-UTI é definida como uma fraqueza muscular generalizada desenvolvida no curso da estadia na UTI a qual a causa não pode ser atribuída além da doença crítica e seu tratamento (33). É datado de 1882 o primeiro relato de FA-UTI onde foi caracterizada pela perda de força e massa muscular em pacientes sob risco de morte (34). Entretanto, somente um século depois Bolton e col. (35) realizaram estudo com biópsia e análise eletrofisiológica o que proporcionou melhor entendimento da doença. Estes autores introduziram o termo polineuropatia da doença crítica após detectarem alterações morfológicas e eletrofisiológicas que caracterizam esta doença. A polineuropatia da doença 
crítica foi definida como uma degeneração axonal primária distal de ambas as fibras (motora e sensitiva) e foi diretamente atribuída ao efeito tóxico da sepse (35).

Dois anos depois foram detectadas mais alterações, além das descritas por Bolton e colaboradores (35), sendo proposta a definição de outra disfunção denominada miopatia da doença crítica (36). Foi demonstrado que esta miopatia primária poderia levar a vários padrões morfológicos e foi presumido ser desencadeada também pela sepse e por outros fatores como $\mathrm{o}$ uso de corticosteroides e bloqueadores neuromusculares $(37,38)$.

Até pouco tempo ainda permanecia a discussão se a polineuropatia e a miopatia da doença crítica seriam desordens distintas com diferentes fisiopatologias ou se estas meramente representariam manifestações em diferentes sistemas (muscular e nervoso) de um mecanismo fisiopatológico comum (39). Alguns estudos sugerem que ambas coexistem e tal condição pode ser denominada polineuromiopatia da doença crítica $(32,38)$. Atualmente, o termo FA-UTI vem sendo considerado o mais adequado para descrever tal doença $(4,40,41)$.

As causas da FA-UTI ainda não são completamente explicadas (40). Tem sido hipotetizado que a resposta do organismo à síndrome da resposta inflamatória sistêmica (SIRS), à sepse e à disfunção de múltiplos órgãos pode estar envolvida neste mecanismo (42-44). A fisiopatologia desta doença pode ser atribuída às alterações, evocadas pela sepse, nas estruturas microvasculares que nutrem os neurônios (45). Estas levam ao aumento da permeabilidade vascular permitindo a penetração de substancias tóxicas na parte distal dos nervos (37). O edema resultante do aumento da permeabilidade neural pode prejudicar o fornecimento de energia para o axônio levando a morte axonal (4). Efeitos tóxicos diretos e disfunção mitocondrial evocados por hiperglicemia podem contribuir para este processo também (46).

Além da sepse e da disfunção de múltiplos órgãos foram identificados outros fatores de risco independentes para o desenvolvimento da FA-UTI, tais como o uso de drogas vasopressoras $(47,48)$ e os antibióticos 
aminoglicosídeos (44). O risco associado a uma maior gravidade da doença, que se reflete pelo escore de gravidade APACHE II (Acute Physiology and Chronic Health disease Classification System) foi demonstrado em vários estudos $(44,49,50)$. A hiperglicemia também foi considerada um fator de risco independente para a FA-UTI e o controle glicêmico por meio de infusão de insulina reduziu a incidência desta doença (51). Além disso, outros fatores como desnutrição e imobilidade no leito também vêm sendo considerados (52). Desta forma, fica claro que, os pacientes com TCE ventilados mecanicamente, são grandes candidatos a desenvolverem a FAUTI.

As estimativas apontam para uma incidência de 26 a $65 \%$ de FA-UTI em pacientes tratados na UTI por sete dias ou mais $(37,38)$ Nas subpopulações com sepse e disfunção de múltiplos órgãos, este valor pode alcançar 100\% (55). As características clínicas frequentes consistem na dificuldade do desmame da ventilação mecânica, tetraparesia com perda de massa muscular e redução ou ausência dos reflexos tendíneos (56). Os músculos faciais são relativamente poupados na FA-UTI, portanto alterações nos nervos cranianos guiam o examinador para outras doenças neurológicas (57). As anormalidades são geralmente marcantes nos membros inferiores do que nos membros superiores e apresentam um padrão tempo de internação dependente (37).

A suspeita da FA-UTI comumente é levantada por meio destas características clínicas, porém a confirmação da doença ocorre com a realização da eletromiografia com eletrodo de agulha e/ou biópsia neuromuscular $(6,38,58)$ os quais são considerados padrão ouro. Contudo, a realização destes exames na UTI raramente é possível, tanto pelo custo operacional, quanto pelo risco imposto aos pacientes.

\subsection{Avaliação eletrofisiológia do doente crítico}

Pacientes criticamente enfermos apresentam alta incidência de alterações eletrofisiológicas (58). A presença destas alterações está fortemente associada a piores desfechos clínicos e funcionais (4). Para a análise eletrofisiológica das estruturas neuromusculares, a eletromiografia 
com eletrodo de agulha é o exame considerado padrão ouro. Estudos prospectivos com pacientes admitidos por várias razões, especialmente sepse, síndrome da resposta inflamatória sistêmica e falência de múltiplos órgãos, demonstraram que 25 a 40\% apresentavam evidencias clínicas de desordem neuromuscular (48,58-63). Coakley e colaboradores.(52) demonstram que a incidência da FA-UTI pode chegar até $90 \%$ quando 0 diagnóstico é baseado em achados eletrofisiológicos. Desde 1984, quando os primeiros exames de eletrodiagnóstico foram empregados na unidade de terapia intensiva (35), parâmetros como o PAMC e o PANS vêm sendo utilizados como marcadores importantes. Apesar de não ser uma rotina nas UTIs, os protocolos para a realização destes testes, bem como a mensuração das alterações eletrofisiológicas em doentes críticos, está bem estabelecidas na literatura, $(6,58)$.

Em pacientes com FA-UTI, com predominância de alterações musculares, os achados mais comuns nos estudos de condução nervosa (ECN) são a redução na amplitude e o aumento na duração do PAMC. Nos pacientes com predominância de alterações axonais os achados eletrofisiológicos mais comuns são a redução nas amplitudes, tanto do PAMC, quanto do PANS sem alteração na velocidade de condução (6). Em estudos de séries de casos com miopatia da doença crítica comprovada por biópsia, mais de $87 \%$ dos pacientes tinham pelo menos um sítio com a amplitude do PAMC diminuída. Normalmente os parâmetros eletrofisiológicos são mensurados nos membros superiores e inferior para testar o PAMC e o PANS dos nervos ulnar, radial, mediano, sural, tibial e fibular por exemplo $(58,64,65)$. Tipicamente mais de um PAMC apresenta-se com a amplitude reduzida e as reduções são geralmente mais de 50\% abaixo do limite inferior de normalidade. Além disso, em alguns pacientes, não só a amplitude está reduzida, mas a duração do PAMC também apresenta-se aumentada.(66-69). Este aumento na duração do PAMC pode estar presente em múltiplos nervos, mas em graus variados e ocorre devido a diminuição na velocidade de condução da fibra muscular e pela redução da excitabilidade da membrana sarcolemal (6). É difícil determinar quando as alterações eletrofisiológicas são puramente causadas pela miopatia ou 
pela polineuropatia da doença crítica, mas tipicamente estas alterações ocorrem entre a primeira e segunda semana no curso da doença crítica $(58,59)$.

Em alguns estudos as anormalidades nos ECN foram encontradas mais precocemente, a partir de 72 horas de admissão na UTI. (6,59). Numa pesquisa com 48 pacientes sépticos submetidos ao ECN a partir de 72 horas de admissão, foram reportadas baixas amplitudes no PAMC em 9 indivíduos (19\%) e redução em ambos PAMC e PANS ou apenas no PANS em 22 (28\%). Um total de $72 \%$ dos pacientes tiveram declínio dos PAMC no decorrer de 7 dias e este declínio predisse fraqueza neuromuscular (59). Resultados similares foram encontrados por outros pesquisadores (58).

No estudo CRIMYNE (58), 92 pacientes admitidos na UTI por diversas etiologias foram submetidos a ECN nas primeiras 24 horas de internação. Esta pesquisa identificou $30,4 \%$ da amostra com polineuropatia axonal, miopatia ou ambas. Os testes de condução nervosa foram realizados diariamente e as primeiras alterações apareceram por volta do sexto dia. Todos os pacientes afetados desenvolveram alterações eletrofisiológicas até o $13^{\circ}$ dia do estudo (58). A redução na amplitude do PAMC em pacientes críticos é um preditor para o desenvolvimento da FA-UTI $(6,58,64)$. A redução na amplitude do PAMC abaixo de dois desvios padrão da normalidade, evocado pelo estímulo do nervo fibular, identifica de forma acurada pacientes com FA-UTI e pode ser utilizado como teste de triagem (6).

Comumente a realização da eletromiografia não está disponível na UTI (70). Isso ocorre tanto pela ausência de equipamentos, quanto pela falta de profissionais especializados na realização e interpretação deste exame. Além disso, é um procedimento invasivo e apresenta inúmeras dificuldades técnicas para ser realizado na UTI. Todos estes empecilhos dificultam a implantação de um protocolo de monitorização dos distúrbios neuromusculares em doentes críticos.

Alternativamente, as alterações na excitabilidade neuromuscular podem ser detectadas por meio do teste de eletrodiagnóstico de estímulo. $O$ eletrodiagnóstico de estímulo é um exame não-invasivo composto pela 
mensuração da reobase, cronaxia e índice de acomodação. A partir da análise destes parâmetros fisiológicos é possível determinar a presença de disfunção na excitabilidade neuromuscular $(7,8,10,71)$, bem como fornecer parâmetros adequados para o tratamento neuromuscular por meio da estimulação elétrica (16).

A EENM é uma alternativa para o tratamento dos pacientes criticamente enfermos restritos ao leito, pois permite a contração passiva da musculatura esquelética, por meio de pulsos elétricos aplicados através da pele a partir de eletrodos de superfície (11). Este tratamento não depende da cooperação do paciente, podendo ser iniciado precocemente, mesmo em pacientes sedados $(72,73)$. Várias pesquisas demonstraram resultados positivos com a utilização da EENM em pacientes críticos como: redução da perda de massa muscular, ganho de força muscular, diminuição na incidência de FA-UTI e aumento das taxas de sucesso no desmame (7276). Os resultados deste tipo de tratamento ainda são incipientes e controversos $(13,14)$, a recomendação por protocolos de EENM mais eficazes vem sendo intensificada $(11,15)$. Uma das principais recomendações está na determinação do ponto motor e na utilização de larguras de pulso superiores ao limiar de excitabilidade neuromuscular (15). Em pacientes com doenças neuromusculares este limiar encontra-se significativamente elevado alcançando valores superiores a $10.000 \mu \mathrm{s}$ $(7,10)$.

A FA-UTI é caracterizada por alterações da excitabilidade neuromuscular com diminuição de força global que podem estar acompanhadas com perda de massa muscular. Compreender e mensurar as alterações eletrofisiológicas neuromusculares nos doentes críticos pode ser a chave para a aplicação de protocolos de EENM mais eficazes $(16,71,77,78)$. Assim, uma vantagem importante do eletrodiagnóstico de estímulo sobre a eletromiografia com eletrodo de agulha está no fato de que somente este primeiro exame é capaz de detectar o ponto motor e estabelecer o limiar de excitabilidade guiando o tratamento com EENM.

O eletrodiagnóstico de estímulo se baseia no princípio diagnóstico das diferentes respostas evocadas pelo nervo e pelo músculo com parâmetros 
de estimulação predeterminados $(7,10)$. Para evocar a contração muscular é necessária a excitação do neurônio adjacente. O estímulo mínimo com forma de pulso retangular e largura de pulso considerada infinita (1000 ms) para alcançar este limiar de excitabilidade é chamado de reobase (79). Já a cronaxia, é a menor largura de pulso para provocar contração visível com o dobro da reobase utilizando um pulso retangular (79). Após a denervação, os valores de cronaxia se tornam maiores, uma vez que estes não são mais adquiridos a partir da resposta do nervo, mas sim, do músculo denervado (8). Nos músculos saudáveis, suas respectivas fibras nervosas disparam à estimulação elétrica, enquanto que nos músculos denervados são as fibras musculares que respondem ao estímulo. Os nervos apresentam menores valores de cronaxia do que as fibras musculares, portanto, músculos inervados têm menores cronaxias (7). O valor médio de cronaxia de um músculo inervado é cerca de $400 \mu$ s (80). O índice de acomodação é a relação entre a acomodação e a reobase. Acomodação é a propriedade que o músculo sadio tem de não responder, ou de responder apenas com altas intensidades, aos pulsos de crescimento lento (exponencial). É a menor intensidade necessária para produzir uma contração muscular, evocada por um pulso exponencial de largura infinita (1000 ms) $(7,16)$. A acomodação expõe as diferentes respostas de nervos e músculos aos pulsos elétricos em formato exponencial. Nas fibras nervosas, um pulso elétrico com formato exponencial, de até duas vezes o valor da reobase, inativa temporariamente os canais de sódio antes da despolarização ocorrer, assim, não é evocada qualquer contração. No caso das fibras musculares, a condutância de sódio é menos alterada por pulsos exponenciais. Desta forma, é possível evocar contrações musculares com pulsos exponenciais de intensidade menor do que o dobro da reobase (7).

Pacientes com valores de cronaxia > $1000 \mu$ s e índice de acomodação < 2 são diagnosticados com alteração da excitabilidade neuromuscular, com sensibilidade de até $100 \%$ (7). Considerando que FAUTI é caracterizada pela disfunção da excitabilidade eléctrica neuromuscular (6), o eletrodiagnóstico de estímulo pode ser uma ferramenta útil para o rastreamento desta doença $(7,8,10)$. 


\subsection{Avaliação da estrutura muscular por ultrassonografia}

Tradicionalmente a FA-UTI é diagnosticada por meio da escala de força volitiva do Medical Research Council (48). Contudo, muitas vezes, ocorre um atraso no diagnóstico da FA-UTI devido ao nível de consciência inadequado dos pacientes para a realização deste tipo de teste (81). Intervenções como biópsia muscular e testes de condução nervosa são usados para identificar indivíduos com FA-UTI $(58,82)$. No entanto, estas condutas são invasivas, caras, nem sempre estão disponíveis na UTI e exigem a presença de profissionais especializados (70). A avaliação muscular por ultrassonografia é um método não volicional e promissor que permite a identificação de alterações na estrutura e morfologia muscular $(70,82,83)$. A ultrassonografia pode ser realizada à beira do leito, não é invasiva, apresenta boa confiabilidade inter e intra-observador $(84,85)$, e está prontamente disponível na maioria dos hospitais (86).

A perda de massa muscular é um problema frequente encontrado nos pacientes internados na UTI (87). Esta perda é muitas vezes mascarada pela retenção de líquidos. Por isso, os métodos antropométricos normais de avaliação das alterações na massa muscular e composição corporal não são aplicáveis (88). Os principais exames de imagem para avaliar a massa muscular de pacientes internados na UTI são a ressonância magnética, a tomografia computadorizada e a ultrassonografia $(89,90)$. Estudos compararam estas técnicas e não encontraram diferenças significativas entre elas $(89,90)$. Assim, em pacientes criticamente enfermos, a ultrassonografia modo- $B$ tem sido a técnica de escolha apresentando-se mais viável e factível do que a tomografia computadorizada e a ressonância magnética $(70,82,87)$.

Para identificar as alterações na estrutura e morfologia muscular nos pacientes internados na UTI, dois parâmetros da ultrassonografia modo B vêm sendo mensurados: a espessura e a ecogenicidade muscular $(70,82,91)$. A espessura e ecogenicidade normalmente são medidas em cortes transversais e em áreas predeterminadas as quais são mantidas ao longo da internação $(41,70,82,83)$. A confiabilidade da medida de espessura e de ecogenicidade do músculo quadríceps foi demonstrada recentemente 
em indivíduos criticamente enfermos (83,92). Sarwal e colaboradores. demonstraram excelente confiabilidade inter e intra-examinador para a avaliação da ecogenicidade da musculatura do quadríceps, independentemente do nível de especialização dos profissionais (92). Com as medidas de espessura e ecogenicidade é possível prever alterações na força muscular e na funcionalidade $(70,87)$. Existe uma relação comprovada entre força e espessura do músculo quadríceps femoral em humanos $(93,94)$. Além disso, foi demonstrado que a diminuição da espessura do músculo quadríceps femoral apresenta correlação com o tempo de permanência na UTI (87). Dessa forma, o exame pode fornecer subsídios para identificar potenciais respondedores à reabilitação ainda na fase precoce da internação (70). Isso é particularmente valoroso, uma vez que os recursos físicos e humanos normalmente são limitados para a realização deste tipo de tratamento (70). 


\section{OBJETIVO}

Descrever as alterações de excitabilidade neuromuscular e da estrutura muscular (espessura e ecogenicidade) em pacientes com TCE submetidos à ventilação mecânica nos primeiros 14 dias de internação na UTI. 


\section{MATERIAIS E MÉTODOS}

\subsection{Desenho do estudo}

Foi realizado um estudo prospectivo observacional para investigar a excitabilidade neuromuscular e a estrutura muscular de pacientes com TCE ventilados mecanicamente durante quatorze dias de internação na UTI (Figura 1). O estudo foi conduzido num hospital público de referência em trauma no Distrito Federal, Brasil, entre maio de 2014 e junho de 2015. O estudo foi aprovado pelo comitê de ética em pesquisa da Fundação de Ensino e Pesquisa em Ciências da Saúde (FEPECS), $n{ }^{\circ}$ 328675, e foi registrado no Australian New Zealand Clinical Trials Registry (ACTRN12614001059651). O termo de consentimento informado foi obtido de um parente próximo, pois todos os pacientes encontravam-se sedados ou não cooperativos.

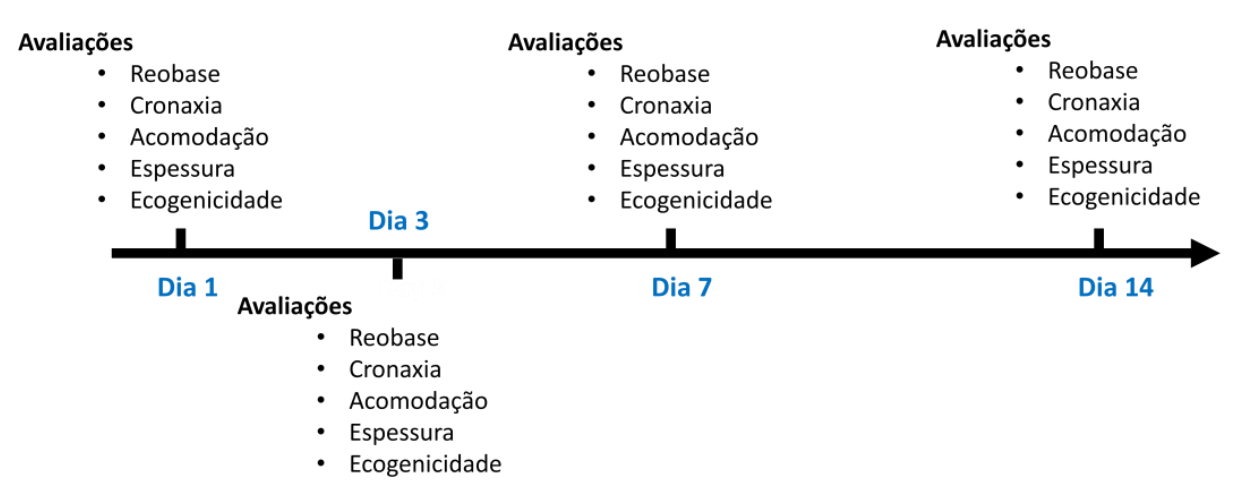

Figura 1 - Desenho do estudo e descrição das avaliações realizadas. Os pacientes foram admitidos no estudo com até 24 horas de internação hospitalar sendo avaliados em quatro momentos: na admissão, no terceiro, sétimo e décimo quarto dia.

\subsection{Pacientes}

Todos os pacientes com TCE internados na UTI, com 18-60 anos de idade em ventilação mecânica foram incluídos no estudo. Os critérios de exclusão foram: fraturas nos membros inferiores, superiores e/ou na coluna vertebral, e lesões em pele no sitio de avaliação. 


\subsection{Avaliações}

Até as primeiras 24 horas de ventilação mecânica na UTI (dia 1), os pacientes foram incluídos no estudo, submetido ao teste de eletrodiagnóstico de estímulo, e avaliação ultrassonográfica (espessura e ecogenicidade muscular). Todas as avaliações foram repetidas além do dia 1, nos dias 3,7 e 14 (Figura 1).

\subsubsection{Teste de eletrodiagnóstico de estímulo.}

A excitabilidade neuromuscular foi conduzida por um gerador de pulso universal, Dualpex 071 (Quark Medical LTDA, Piracicaba, Brasil). Para a medição da reobase, a corrente foi aumentada de 0 para $69 \mathrm{~mA}$ em incrementos de $1 \mathrm{~mA}$ até que houvesse uma ligeira, mas claramente visível, contração muscular. A avaliação foi realizada com pulso retangular, com duração de 1 segundo e intervalo de 2 segundos (7). Para a medição da cronaxia, a largura de pulso foi aumentada a partir de $20 \mu$ s até $1 \mathrm{~ms}$ em incrementos de $100 \mu \mathrm{S}$. A partir de $1 \mathrm{~ms}$, incrementos de $1 \mathrm{~ms}$ foram realizados com uma intensidade de corrente duas vezes o valor da reobase até que houvesse uma leve, mas visível contração muscular (7). Para medições da acomodação, a corrente foi aumentada de 0 a $69 \mathrm{~mA}$ em incrementos de $1 \mathrm{~mA}$ até que ocorresse uma leve, mas claramente visível contração muscular. As medidas de acomodação foram executadas com pulso em formato exponencial com 1 segundo de duração e intervalo de 2 segundos (7).

O índice de acomodação foi determinado pela divisão dos valores de acomodação pela reobase. [Índice de acomodação $=$ (acomodação $/$ reobase)] Um índice acomodação abaixo de 2, associado a uma cronaxia > 1000 us aumenta a sensibilidade do teste de eletrodiagnóstico de estímulo para determinação de disfunção na excitabilidade neuromuscular (7). O teste de eletrodiagnóstico de estímulo foi realizado em três músculos: bíceps BB, RF e TA. Um eletrodo de referência (polo positivo) com $100 \mathrm{~cm}^{2}$ de área foi colocado no tornozelo para todas as medições e o eletrodo ativo (polo negativo) em formato de caneta com $1 \mathrm{~cm}^{2}$ de área foi usado para encontrar os pontos motores e determinar os valores de reobase, cronaxia e 
acomodação nestes. A área de varredura foi estabelecida com base em publicações prévias $(95,96)$.

O ponto motor do músculo representa o local no qual o ramo motor do nervo penetra no ventre muscular. Assim, este é o ponto ideal para medir a excitabilidade neuromuscular. A localização do ponto motor foi marcada com caneta dermográfica de alta fixação para evitar diferentes posições ao longo dos dias.

\subsubsection{Ultrassonografia}

A espessura e ecogenicidade muscular foram medidas utilizando um dispositivo de ultrassonografia portátil em modo B, M-Turbo® (Sonosite, Bothwell, WA, EUA). A avaliação da espessura do músculo, com ultrassonografia é uma alternativa válida e confiável para medir a estrutura muscular. A diminuição da espessura muscular expressa atrofia (86). Um gel de transmissão solúvel em água foi aplicado ao local de medição e um transdutor linear de 7,5 MHz foi posicionado perpendicular à interface do tecido com a menor compressão possível da pele. A espessura muscular foi mensurada em três músculos: BB, RF e TA. O transdutor foi posicionado de acordo com uma recomendação anterior (97). O BB foi avaliado no 2/3 distal da distância entre o acrômio e a fossa cubital. A avaliação do RF foi conduzida na distância média entre a espinha ilíaca anterossuperior e borda superior da patela. O TA foi avaliado no 1/4 proximal da distância entre a borda inferior da patela e o maléolo lateral. As medidas foram realizadas sempre ao longo dos dias no mesmo local predefinido.

Após a aquisição das imagens a avaliação da espessura foi realizada de acordo com as recomendações de Arts e colaboradores (97) O BB (incluindo o músculo braquial subjacente) foi medido entre a parte superior do úmero e da fáscia superficial do bíceps (Figura 2A); o RF (incluindo o vasto intermédio) foi medido entre a parte superior do fêmur e da fáscia superficial do reto femoral (Figura 2B); e o TA que foi medido entre a membrana interóssea (ao lado da tíbia) e a fáscia superficial do tibial anterior (Figura 2C). 

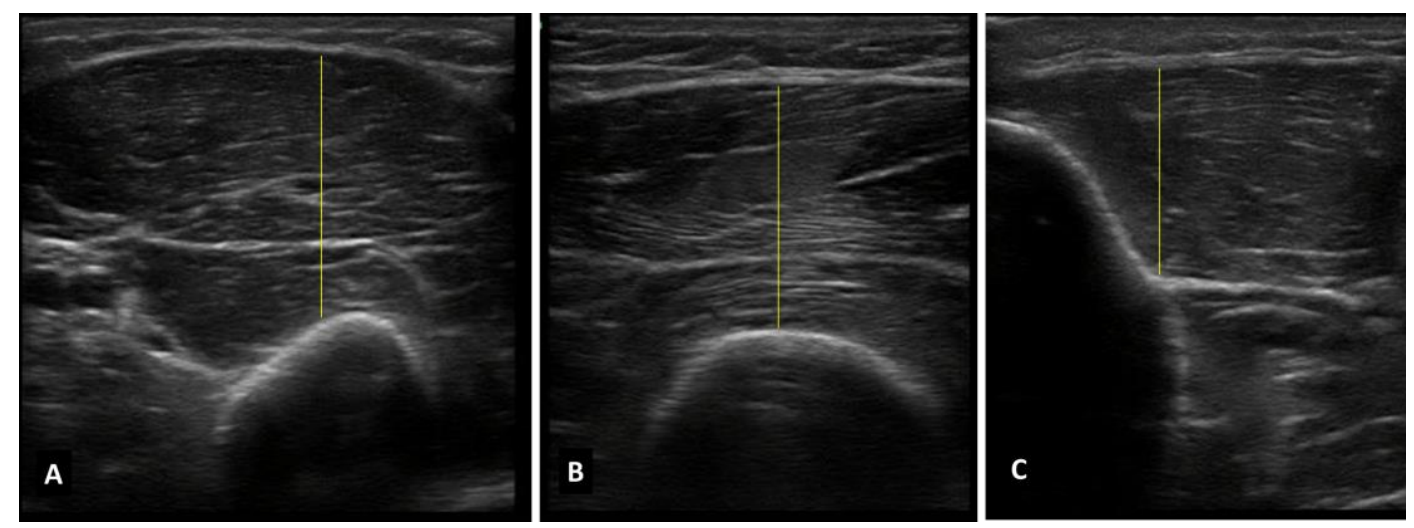

Figura 2 - Locais de mensuração da espessura muscular. Bíceps braquial (A); reto femoral (B); tibial anterior (C) após a aquisição das imagens segundo as recomendações de Arts e colaboradores (97).

Todos os pontos foram marcados com tinta para evitar a substituição ao longo dos dias. A espessura e a ecogenicidade muscular foram analisadas com o software ImageJ (http://imagej.nih.gov/ij/) (98). A ecogenicidade muscular vem sendo mensurada nas imagens por meio da analise da escala de cinza. Os músculos afetados aparecem mais brancos (ou seja, têm um aumento na ecogenicidade). Esta maior ecogenicidade é resultado do aumento de tecido fibroso e adiposo intramuscular além de zonas de necrose $(82,99)$. A região de interesse para mensuração da ecogenicidade foi selecionada em cada músculo para incluir o máximo de área muscular possível, sem qualquer osso ou fáscia circundante. A ecogenicidade em cada região de interesse foi calculada pela média de três medidas por músculo (100). Com uma resolução de 8 bits as imagens resultam em valores que variam entre 0 (preto) e 255 (branco).

No braço, somente o BB foi incluído para avaliação da ecogenicidade. No quadríceps femoral, o músculo RF foi utilizado para análise por conta de uma limitação técnica que dificultou medir com precisão o vasto intermédio. Todas as análises (espessura e ecogenicidade) foram realizadas nas três melhores imagens adquiridas, usando software ImageJ. A espessura 
muscular foi medida em centímetros no ponto mais largo do músculo. Foram utilizadas as médias dos valores encontrados nas três imagens. Um fisioterapeuta treinado realizou todas as avaliações com coeficiente de correlação intraclasse de 0,99 e 0,98 para espessura e ecogenicidade respectivamente.

\subsection{Análise estatística}

A normalidade dos dados foi testada com o teste de Shapiro Wilk e as variáveis paramétricas foram descritas em média e desvio padrão ( $\pm D P$ ). Variáveis não paramétricas foram apresentadas em mediana e IIQ. As diferenças nas cronaxias em cada músculo ao longo dos dias foram medidas pelo teste de Friedman ajustado para comparações múltiplas pelo teste de Dunn.

Para avaliar as variáveis categóricas (presença ou não de disfunção na excitabilidade neuromuscular, determinado pela presença de cronaxia > 1000 us e índice de acomodação < 2), foi utilizado o teste McNemar. Diferenças na espessura muscular e na ecogenicidade em cada musculo ao longo dos dias foram medidas pelo teste de analise de variância (ANOVA) de medidas repetidas ajustada para comparações múltiplas pelo teste post hoc de Tukey. O Teste ANOVA One Way foi utilizado para comparar a diminuição da espessura entre os três músculos com o post hoc de Tukey. Homogeneidade foi medida com o teste de Levene, e a esfericidade dos dados foi testada pelo teste de esfericidade de Mauchly com correção de Greenhouse-Geisser Diferença estatisticamente significante foi considerada quando $p<0,05$. Os dados foram analisados usando o software Graphpad Prism 6. 


\section{RESULTADOS}

\subsection{Características de base e fluxo do estudo}

Cento e quarenta e sete pacientes foram potencialmente elegíveis para o estudo, sendo quarenta e nove incluídos. Noventa e oito pacientes foram excluídos devido a: fraturas nos membros e/ou coluna vertebral, lesões em pele, morte encefálica, ou por recusa na assinatura do termo consentimento. No dia 3, havia 41 pacientes e até o sétimo dia ocorreram 10 perdas (7 óbitos e 3 altas). No dia 7 havia 31 pacientes e até o decimo quarto dia ocorreram 9 perdas (1 óbito e 8 altas). No dia 14, restaram 22 pacientes os quais foram analisados. O diagrama de fluxo está apresentado na (Figura 3). As características de base dos pacientes são demonstradas na tabela 1 , com média de idade de 39 ( \pm 3) anos de idade, $82 \%$ dos pacientes do sexo masculino com mediana do APACHE II de 14 IIQ (10-18).

Tabela 1: Características de base

\begin{tabular}{|c|c|}
\hline Diagnóstico principal, n (\%) & TCE, $22(100 \%)$ \\
\hline Gênero masculino, n (\%) & $18(82 \%)$ \\
\hline Idade, anos (media \pm DP) & $39( \pm 3)$ \\
\hline APACHE II (0-70) (mediana IIQ) & $14(10-18)$ \\
\hline Duração da sedação, dias (media \pm DP) & $12.25( \pm 4,4)$ \\
\hline Uso de corticoide, dias (media \pm DP) & 0 \\
\hline Uso de bloqueador neuromuscular, dias (media \pm DP) & 0 \\
\hline Uso de aminoglicosídeos, dias (media \pm DP) & 0 \\
\hline Uso de vasopressor, dias (media \pm DP) & $6,3( \pm 3,8)$ \\
\hline \multicolumn{2}{|l|}{ Desfechos: } \\
\hline \multicolumn{2}{|l|}{ - Dias em VM (media \pm DP) } \\
\hline - Tempo de permanência na UTI, dias (media \pm DP) & $22.5( \pm 6,1)$ \\
\hline - $\quad$ Alta da UTI, n (\%) & $22(100 \%)$ \\
\hline - $\quad$ Mortalidade na UTI, n (\%) & $0(0 \%)$ \\
\hline
\end{tabular}

APACHE II: Acute Physiology and Chronic Health disease Classification System II; DP: desvio padrão; IIQ: intervalo entre quartis; TCE: traumatismo cranioencefálico; UTI: unidade de terapia intensiva; VM: ventilação mecânica. 


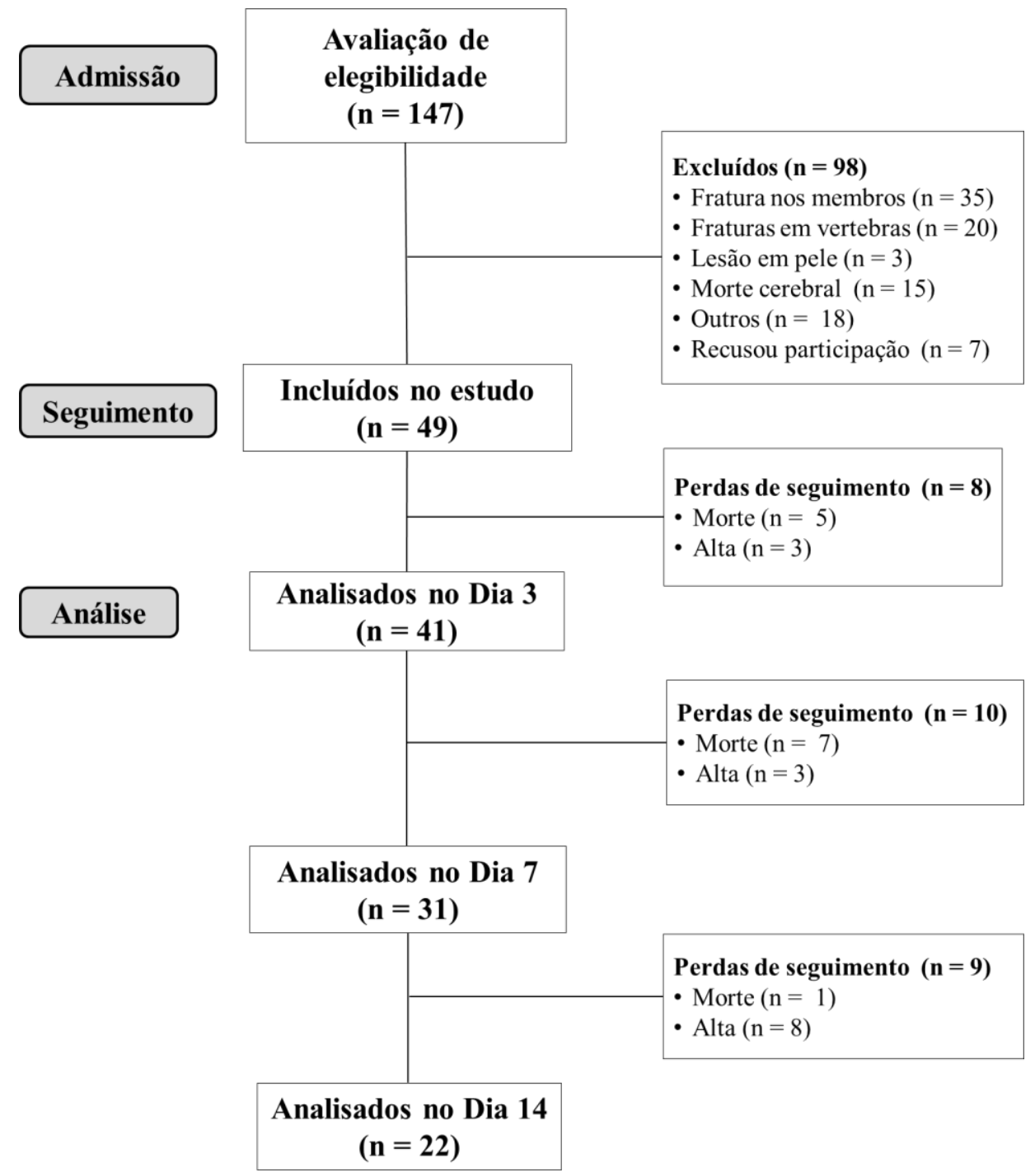

Figura 3 - Diagrama de fluxo

\subsection{Dados eletrofisiológicos}

A reobase mediana ao longo dos dias foi de 6 (4-8) mA para TA, 16 (12-24) mA para RF e 5 (3-8) mA para o BB. O comportamento da cronaxia ao longo dos dias para cada músculo é apresentado na figura 4. 


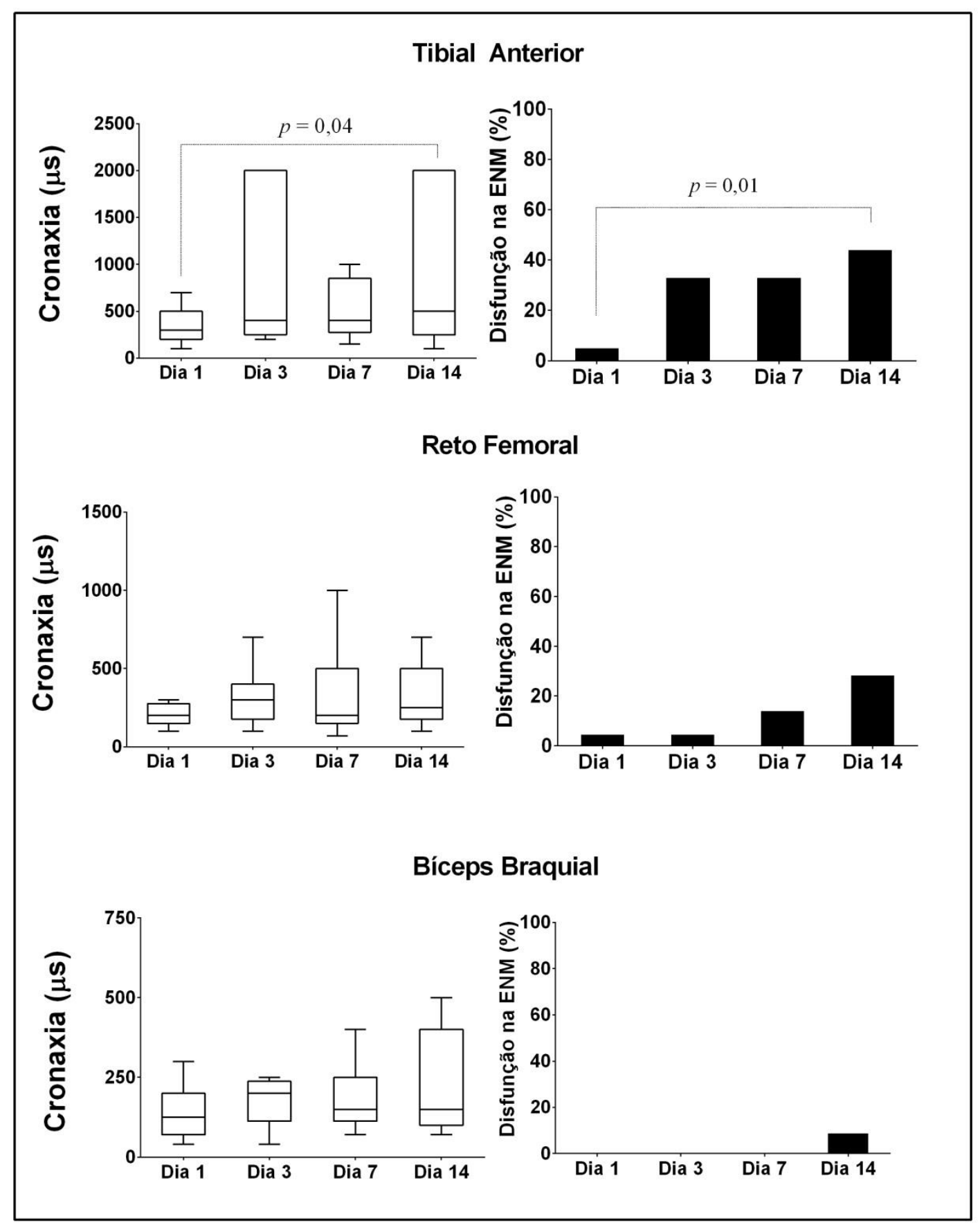

Figura 4 - Avaliação da excitabilidade neuromuscular. ENM: excitabilidade neuromuscular. A análise da disfunção da excitabilidade neuromuscular foi determinada pela presença de cronaxia $>1000 \mu \mathrm{s}$ e índice de acomodação $<2$.

Diferenças estatisticamente significativas foram detectadas apenas no TA quando se comparou a mediana do dia 1,300 (200-700) $\mu$ s vs. dia 14, 500 (250-2000 ) $\mu \mathrm{s}, p=0,04$. Não foi observada diferença no RF entre o dia $1,200(150-275) \mu s$ e dia 14, $250(175-500) \mu s, p>0,99$. O BB teve uma 


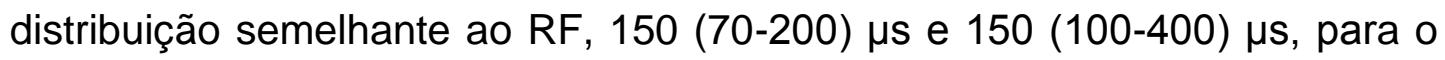
dia 1 e o dia 14, respectivamente, $p>0,99$. A definição de disfunção na excitabilidade neuromuscular foi dicotomicamente estabelecida pela presença de cronaxia $>1000 \mu$ s além do índice de acomodação $<2$. A acomodação mediana ao longo dos dias foi de 6 (1-11) mA para TA, 21 (834) $\mathrm{mA}$ para RF e 9 (2-16) $\mathrm{mA}$ para o BB. O acompanhamento da excitabilidade neuromuscular do dia 1 ao dia 14 mostrou um aumento na taxa absoluta de disfunção da excitabilidade neuromuscular para todos os músculos: 44\% no TA; $29 \%$ no RF e $9 \%$ no BB. No entanto, este aumento só foi estatisticamente significativo no TA, $p=0,03$. Estes dados são apresentados na Figura 4

\subsection{Estrutura muscular}

A espessura muscular e a ecogenicidade ao longo dos dias para cada músculo são demonstrados na figura 5. Uma redução estatisticamente significativa foi detectada no TA quando se comparou a espessura no dia 1 , $2,47( \pm 0,46) \mathrm{cm}$ vs. dia $7,2,42( \pm 0,36) \mathrm{cm}, \mathrm{p}=0,006$ e no dia $1,2,47( \pm$ $0,46) \mathrm{cm}$ vs. dia $14,1,98( \pm 0,28) \mathrm{cm}, \mathrm{p}=0,01$. O mesmo comportamento foi observado na espessura de RF quando se comparou o dia 1, 3,75 $( \pm 0,57)$ $\mathrm{cm}$ vs dia $14,3,14( \pm 0,74) \mathrm{cm}, \mathrm{p}=0,004$. O BB apresentou uma redução estatisticamente significativa na espessura entre o dia $1,3,61( \pm 0,58) \mathrm{cm}$ e dia $14,3,13( \pm 0,45) \mathrm{cm}, p=0,02$. A porcentagem de perda na espessura muscular entre dia 1 e dia 14 em TA, RF e BB foi, respectivamente, 21, 19 e $12 \%$. No entanto, não houve diferença estatística na comparação inter músculos, TA vs RF, $p=0,8$; TA vs $B B, p=0,9$; RF vs $B B, p=0,84$. $O$ musculo TA foi o único a apresentar diferenças estatisticamente significativas na ecogenicidade ao longo dos dias. Estas diferenças foram detectadas entre o dia 1, 56,5 ( $\pm 6,4)$ au vs dia 3, 64,6 ( $\pm 9,5)$ au, $p=0,03$; dia $1,56,5( \pm 6,4)$ au vs dia 7, $66( \pm 11,2)$ au, $p=0,007$ e entre o dia 1, 56,5 $( \pm 6,4)$ au vs dia $14,67,3( \pm 7,6)$ au, $p=0,04$. 


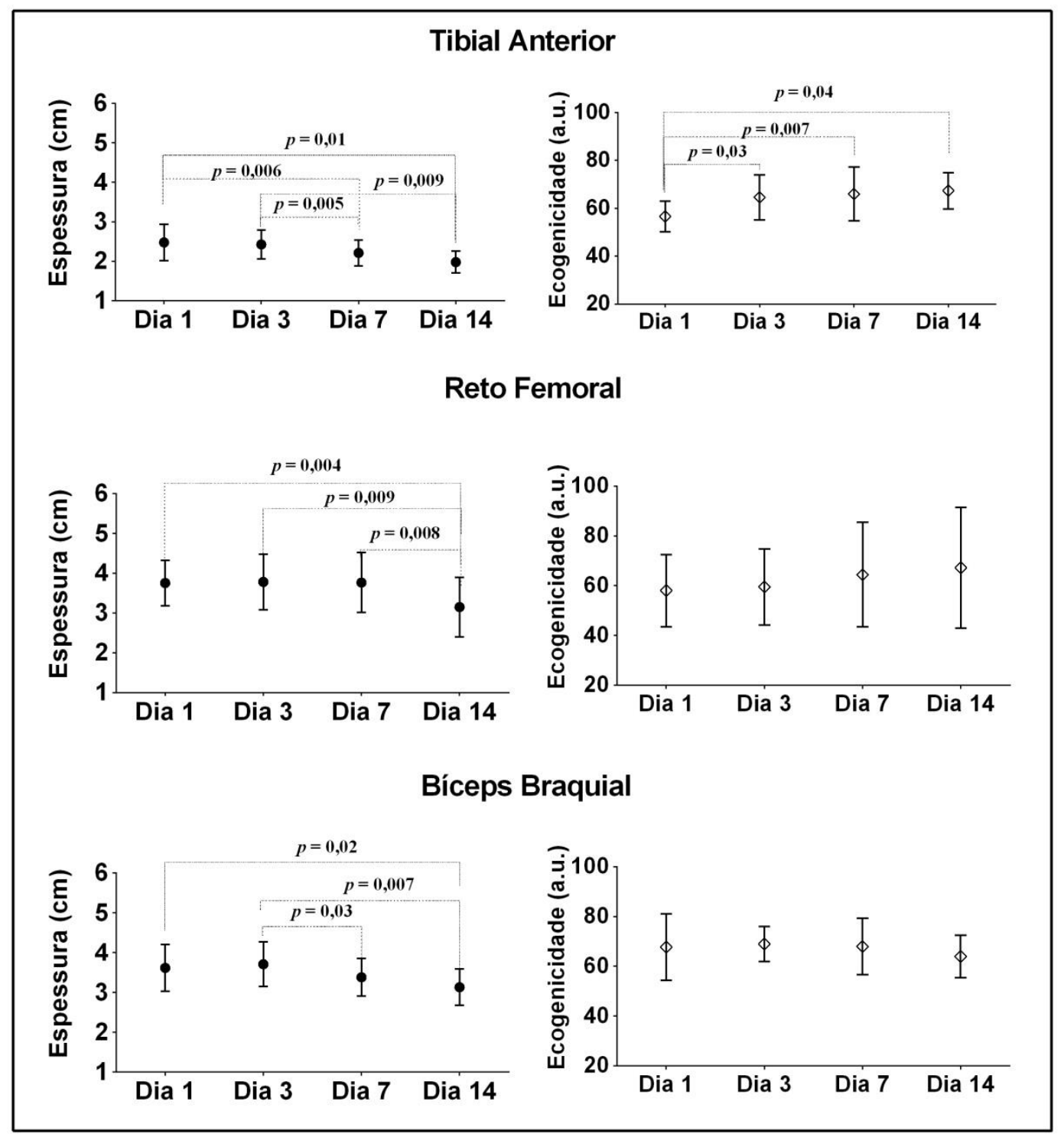

Figura 5 - Avaliação ultrassonográfica da espessura e ecogenicidade muscular. 


\section{DISCUSSÃO}

Os principais resultados deste estudo suportam a ideia de que pacientes com TCE submetidos à VM não só apresentam alterações na estrutura musculoesquelética, mas também evoluem com disfunção da excitabilidade neuromuscular, A utilização do teste de eletrodiagnóstico de estímulo é uma ferramenta viável para medir a disfunção na excitabilidade neuromuscular na UTI e também para oferecer parâmetros que podem melhorar a eficácia dados protocolos de EENM.

Os pacientes com TCE mecanicamente ventilados têm uma perda de massa muscular generalizada, como já demonstrando anteriormente $(5,17)$. Todos os três músculos avaliados apresentaram diminuição estatisticamente significativa na espessura muscular, mas apenas TA desenvolveu disfunção na excitabilidade neuromuscular. Este é um achado relevante que pode impactar diretamente sobre tratamento e nos desfechos funcionais. A taxa de disfunção de excitabilidade neuromuscular alcançou 44\% no TA, contra $29 \%$ e $9 \%$ no RF no BB, respectivamente. Outros estudos demonstraram maior incidência de axonopatia no nervo fibular, porém essas modificações foram avaliadas por meio de eletromiografia com eletrodo de agulha e biópsia muscular $(38,58,101)$. As alterações de excitabilidade do nervo fibular parecem ser inevitáveis nos pacientes com FA-UTI (58). Uma possível explicação para esse fenômeno está relacionada com o seu comprimento, pois o nervo fibular é mais longo que outros nervos do corpo tornando-se mais vulnerável ao déficit de energia causado por isquemia ou disóxia do tecido (58). A hipótese da falha bioenergética pode explicar a maior incidência de neuropatia no nervo fibular (58). A geração do potencial de ação neural e a integridade estrutural distal do axônio são criticamente dependentes do transporte axonal de proteínas e outras moléculas (102). Os axônios não são providos de maquinaria celular para os processos de biossíntese. Todos os nutrientes são sintetizados no corpo do neurônio e em seguida transportados para o seu destino final dentro do axônio (102). Este processo promove um gasto de energético considerável. Quando os neurônios não recebem nutrição adequada devido alterações na 
microcirculação, o transporte axonal pode falhar levando ao aparecimento de axonopatia (103).

É importante enfatizar que a FA-UTI pode ocorrer independentemente da perda de massa muscular e não devem ser consideradas sinônimo $(70,104)$. As alterações musculares esqueléticas foram observadas em pacientes com lesão do motoneurônio superior como os TCE (105). Alterações do motoneurônio inferior devido a lesões neurais centrais podem ser detectadas pela alteração na excitabilidade neuromuscular refletida nos valores cronaxia (8). Diferentes causas estão associadas à rápida perda muscular nesta população, dentre estas o desuso muscular e a ausência da influência trófica do motoneurônio superior (106). A degeneração transneuronal de motoneurônios inferiores após lesão de motoneurônios superiores foi sugerida com base em estudos eletrofisiológicos (106). Portanto, isso pode explicar o aparecimento precoce de disfunção na excitabilidade neuromuscular no TA e RF, detectados um dia após o TCE, 6\% e 5\%, respectivamente. Estes achados têm impacto direto sobre o tratamento das limitações funcionais desenvolvidas na UTI. O entendimento dessas alterações musculares pode afetar os procedimentos de reabilitação. A medição da reobase, cronaxia e acomodação pode melhorar a aplicação da EENM para o tratamento de músculos denervados e dessa forma aumentar a eficácia desta terapêutica (71).

Todos os estudos com EENM na UTI utilizaram valores predeterminados de largura de pulso para todos os pacientes $(11,107,108)$. A largura máxima de pulso utilizada foi $500 \mu \mathrm{s}$, fora da janela terapêutica encontrada no dia 14, no TA dos nossos pacientes, $2000 \mu$ s. Este fato pode explicar algumas dificuldades relatadas por Rodriguez e colaboradores e Segers e colaboradores (107). Rodriguez e colaboradores (73) avaliaram a eficácia de um protocolo de EENM em pacientes sépticos usando uma largura de pulso fixa de $300 \mu \mathrm{s}$. Embora quase a intensidade máxima do equipamento tenha sido utilizada, contrações perceptíveis no bíceps e no quadríceps só foram detectadas em $77 \%$ das sessões. Segers e colaboradores (107) realizaram EENM no quadriceps femoral em doentes críticos e relataram que, em alguns indivíduos com edema significativo, 
nenhuma contração foi detectada durante procedimento. Assim, eles introduziram o conceito de "respondedores ou não respondedores" à EENM, e atribuiram esse fato a presenca de edema. Segers e colaboradores (107), também utilizaram um protocolo de EENM pré-estabelecido onde a excitabilidade neuromuscular não foi determinada. Desta forma, acreditamos que esta seja uma definição inadequada, uma vez que mesmo pacientes com denervação respondem à EENM se a largura de pulso aplicada for maior do que cronaxia e a amplitude de corrente estiver ajustada duas vezes acima da reobase.

Outros autores demonstraram que o edema aumenta a impedância elétrica dos tecidos, aumentando a necessidade de cargas elétricas mais elevadas para produzir a contração muscular (109). Foi demonstrado que estímulos de 50 a 60 mA com $200 \mu$ s de largura de pulso, eram necessários para evocar respostas supra máximas em uma população de pacientes anestesiados (110). Em pacientes criticamente enfermos sem edema periférico, Haper e colaboradores (109) encontraram amplitudes de corrente similares para evocar este nível de resposta. No entanto, a presença de edema, aumentou a necessidade de maiores amplitudes para tanto (109). Haper e colaboradores (109) atribuíram isso à presença de edema o qual diminui a densidade de corrente disponível para a estimulação do nervo e músculo (109). Encontramos uma mediana e $I I Q$ de reobase (estímulo mínimo para gerar contração) de 6 (4-8) mA para TA, 16 (12-24) mA para RF e 5 (3-8) mA para BB.. Portanto, o edema pode ser um obstáculo para a realização da EENM como foi mostrado por Segers e colaboradores (107). Porém, a mensuração da reobase e cronaxia pode superar esta barreira e melhorar a eficácia da EENM.

O teste eletrofisiológico considerado mais adequado para o diagnóstico de doença neuromuscular periférica é a eletromiografia com eletrodo de agulha, mas a sua aplicação na UTI pode ser uma tarefa difícil (6). Essas medidas são invasivas, apresentam alto custo, requerem profissionais especializados e equipamentos de eletromiografia que nem sempre estão disponíveis nas unidades (70). Medidas de excitabilidade nervosa pelo teste eletrodiagnóstico de estímulo fornecem informações 
complementares à avaliação com eletromiografia convencional, $(7,8,80)$. O teste de eletrodiagnóstico de estímulo é um exame não-invasivo e que permite determinar a presença de disfunção na excitabilidade neuromuscular $(7,8,10,71)$. Este tem sido usado para avaliar a atividade elétrica neuromuscular baseando-se no princípio diagnóstico das diferentes respostas evocadas nos nervos e nos músculos por diferentes padrões de estímulo elétrico $(7,39)$. Em músculos inervados, suas respectivas unidades motoras disparam à estimulação elétrica, enquanto que nos músculos denervados, são as fibras musculares que reagem à estimulação elétrica. Nervos tem cronaxias menores do que os músculos, assim, os músculos inervados apresentam menores cronaxias (7). O valor médio de cronaxia num músculo saudável é cerca de $400 \mu$ s (80). O índice de acomodação é a relação entre a acomodação e reobase. A acomodação expõe as diferentes respostas dos nervos e dos músculos à corrente exponencial. Nas fibras nervosas, uma onda com formato exponencial e amplitude até duas vezes a reobase, inativa os canais de sódio antes que a despolarização seja atingida. Assim, não é detectada contração. No caso das fibras musculares, a condutância de sódio é menos alterada pelas correntes exponenciais. Assim, a contração muscular é gerada mesmo com valores de acomodação inferiores a duas vezes a reobase (7). Pacientes com valores de cronaxia maiores do que $1000 \mu \mathrm{s}$, com índice de acomodação inferior a 2, podem ser diagnosticados com disfunção da excitabilidade neuromuscular, com excelente sensibilidade (até 100\%) (7). Considerando que FA-UTI é caracterizada por disfunção na excitabilidade neuromuscular (6), o teste de eletrodiagnóstico de estímulo pode ser uma ferramenta útil na triagem desta doença $(7,8,10)$.

Levando-se em conta os obstáculos para realização da avaliação completa da eletromiografia com eletrodo de agulha, Latronico e colaboradores (101) desenvolveram um teste eletrofisiológico simplificado e não invasivo registrando a velocidade de condição e amplitude dos PAMC evocados pelo estímulo do nervo fibular (denominaram de teste do nervo fibular) (58). Esses autores realizaram um estudo prospectivo, observacional, multicêntrico com cento e vinte e um pacientes para avaliar a 
acurácia deste teste para o diagnóstico da FA-UTI em comparação à eletromiografia clássica. Eles concluíram que o teste do nervo fibular apresentou uma sensibilidade de $100 \%$ e especificidade de $85 \%$, e poderia ser utilizado como um teste de rastreamento para o diagnóstico da FA-UTI. Embora este teste seja viável (tempo médio de execução de 10 minutos) e tenha uma boa precisão, os dispositivos utilizados para tal ainda são caros e não são usuais na UTI. Além disso, o teste do nervo fibular não fornece informações sobre como os parâmetros da EENM devem ser ajustados, uma vez que a cronaxia não pode ser mensurada. Portanto, o teste de eletrodiagnóstico de estímulo é uma ferramenta alternativa não só para o diagnóstico das alterações de excitabilidade neuromuscular, mas também para ajudar nas definições dos parâmetros as serem utilizados na EENM.

A medida da espessura do músculo está intimamente relacionada com a força muscular $(93,94)$. Gruther e colaboradores (87), encontraram associação entre perda de massa muscular no quadríceps femoral e maior tempo de permanência na UTI (87). Nós avaliamos uma população homogênea a qual apresentou padrão similar de perda de massa muscular impossibilitando esse tipo de análise. No entanto, descobrimos que os primeiros dias de internação na UTI são decisivos para o prognóstico de sobrevivência de pacientes com TCE. Nós encontramos maior mortalidade entre os dias 3 e 7. Todos os nossos pacientes que chegaram ao décimo quarto dia de internação, evoluíram com alta para a enfermaria após uma média de 22,5 ( $\pm 6,1)$ dias de permanência na UTI.

Com relação a ecogenicidade, já foi demonstrada a correlação entre o aumento nessa variável e a presença de necrose muscular em pacientes criticamente enfermos (111). Estes achados suportam que a ecogenicidade pode ser um marcador representativo de necrose muscular e destruição da arquitetura ao nível celular (70). Nossos resultados de ecogenicidade apresentaram algumas diferenças dos encontrados por Parry e colaboradores (70). Estes autores demonstraram aumento significativo na ecogenicidade do RF. No presente estudo encontramos aumento da ecogenicidade apenas no TA. Provavelmente, isso aconteceu porque nós avaliamos diferentes populações. Parry e colaboradores estudaram uma 
população heterogênea o que pode gerar alguns fatores confundidores, enquanto nós estudamos apenas pacientes com TCE. Nossos pacientes apresentaram menor pontuação no escore APACHE II do que os pacientes estudados por estes autores. (70). Isso também pode ter levado a uma maior incidência de FA-UTI provocando maior ecogenicidade no RF (83). Além disso, nós definimos uma região de interesse diferente, toda a área visível do músculo. Parry e colaboradores (70) usaram uma região de interesse de $2 \times 2 \mathrm{~cm}$. Em pacientes com padrões heterogêneos a definição da região de interesse pode afetar os resultados encontrados (112).

Interessantemente, Grimm e colaboradores (83) demonstraram a aplicabilidade da ultrassonografia como ferramenta para a diagnóstico da FA-UTI. Neste estudo foi demonstrado que pacientes com alterações eletrofisiológicas, confirmadas por eletromiografia com eletrodo de agulha, exibiam maiores valores de ecogenicidade. Apesar de termos utilizado um teste não invasivo para mensurar as alterações na excitabilidade neuromuscular, os nossos resultados ultrassonográficos de ecogenicidade respaldam estas medidas eletrofisiológicas. O TA foi o único músculo a apresentar alteração da excitabilidade neuromuscular e aumento na ecogenicidade com valores estatisticamente significativos. Estes achados estão em acordo com a teoria da falha energética a qual justifica a maior incidência de alterações no nervo fibular. Finalmente, é importante destacar as limitações desse estudo. Foi registrada a perda de 27 indivíduos devido a alta da unidade ou óbito durante o estudo. Além disso, não foi possível comparar nossos resultados eletrofisiológicos e ultrassonográficos com testes funcionais e de força muscular. O nível de consciência dos nossos pacientes não permitiu que estas avaliações fossem realizados. Novos estudos devem concentrar-se na correlação entre os resultados do teste de eletrodiagnóstico de estímulo e avaliação ultrassonográfica com os desfechos funcionais. Além disso, na eficácia dos protocolos de EENM baseados no limiar de excitabilidade neuromuscular. 


\section{CONCLUSÃO}

Os pacientes com TCE submetidos à ventilação mecânica apresentam não só alteração na estrutura muscular no curso da estadia na UTI, mas também desenvolvem disfunção na excitabilidade neuromuscular. O músculo tibial anterior foi o mais afetado apresentando tanto alterações estruturais quanto eletrofisiológicas. 


\section{REFERÊNCIAS}

1 Sharma, B.; Lawrence, D.W. Top-cited articles in traumatic brain injury. Front Hum Neurosci, v. 8, p. 879, 2014.

2 Peeters, W.; Brande, R. van den; et al. Epidemiology of traumatic brain injury in Europe. Acta Neurochir (Wien), v. 157, n. 10, p. 1683-96, 2015.

3 Wijayatilake, D.S.; Sherren, P.B.; et al. Systemic complications of traumatic brain injury. Curr Opin Anaesthesiol, v. 28, n. 5, p. 525-531, 2015.

4 Hermans, G.; Berghe, G. Van den Clinical review: intensive care unit acquired weakness. Crit Care, v. 19, n. 1, p. 274, 2015.

5 Hirose, T.; Shiozaki, T.; et al. The effect of electrical muscle stimulation on the prevention of disuse muscle atrophy in patients with consciousness disturbance in the intensive care unit. J Crit Care, v. 28, n. 4, p. 536.e1-7, 2013.

6 Lacomis, D. Electrophysiology of neuromuscular disorders in critical illness. Muscle Nerve, v. 47, n. 3, p. 452-63, 2013.

7 Paternostro-Sluga, T.; Schuhfried, O.; et al. Chronaxie and accommodation index in the diagnosis of muscle denervation. Am $\mathbf{J}$ Phys Med Rehabil, v. 81, n. 4, p. 253-60, 2002.

8 Schuhfried, O.; Kollmann, C.; et al. Excitability of chronic hemiparetic muscles: determination of chronaxie values and strength-duration curves and its implication in functional electrical stimulation. IEEE Trans Neural Syst Rehabil Eng, v. 13, n. 1, p. 105-9, 2005.

9 Peviani, S.M.; Russo, T.L.; et al. Stretching and electrical stimulation regulate the metalloproteinase-2 in rat denervated skeletal muscle. Neurol Res, v. 32, n. 8, p. 891-6, 2010.

10 Ervilha, U.; Araujo, R. Estudo sobre a freqüência de distribuição da cronaxia e a sua correlação com distintos graus de lesões nervosas periféricas. Braz J Phys Ther, v. 1, n. 2, p. 45-50, 1996.

11 Maffiuletti, N.A.; Roig, M.; et al. Neuromuscular electrical stimulation for preventing skeletal-muscle weakness and wasting in critically ill patients: a systematic review. BMC Med, v. 11, p. 137, 2013.

12 Agrawal, M.; Joshi, M. Impact of rehabilitation on functional outcome during the first year of moderate and severe traumatic brain injury. Brain Inj, v. 28, n. 3, p. 292-7, 2014.

13 Gruther, W.; Kainberger, F.; et al. Effects of neuromuscular electrical 
stimulation on muscle layer thickness of knee extensor muscles in intensive care unit patients: a pilot study. J Rehabil Med, v. 42, n. 6, p. 593-7, 2010.

14 Poulsen, J.B.; Møller, K.; et al. Effect of transcutaneous electrical muscle stimulation on muscle volume in patients with septic shock. Crit Care Med, v. 39, n. 3, p. 456-61, 2011.

15 Maffiuletti, N.A.; Minetto, M.A.; et al. Electrical stimulation for neuromuscular testing and training: state-of-the art and unresolved issues. Eur J Appl Physiol, v. 111, n. 10, p. 2391-7, 2011.

16 Cummings, J.P. Conservative management of peripheral nerve injuries utilizing selective electrical stimulation of denervated muscle with exponentially progressive current forms. J Orthop Sports Phys Ther, v. 7, n. 1, p. 11-5, 1985.

17 Langlois, J.A.; Rutland-Brown, W.; et al. The epidemiology and impact of traumatic brain injury: a brief overview. J Head Trauma Rehabil, v. 21, n. 5, p. 375-8, 2006.

18 The changing landscape of traumatic brain injury research. Lancet Neurol, v. 11, n. 8, p. 651, 2012.

19 Menon, D.K.; Schwab, K.; et al. Position statement: definition of traumatic brain injury. Arch Phys Med Rehabil, v. 91, n. 11, p. 1637-40, 2010.

20 Roozenbeek, B.; Maas, A.I.R.; et al. Changing patterns in the epidemiology of traumatic brain injury. Nat Rev Neurol, v. 9, n. 4, p. 231-6, 2013.

21 Bruns, J.; Hauser, W.A. The epidemiology of traumatic brain injury: a review. Epilepsia, v. 44 Suppl 1, p. 2-10, 2003.

22 Tagliaferri, F.; Compagnone, C.; et al. A systematic review of brain injury epidemiology in Europe. Acta Neurochir (Wien), v. 148, n. 3, p. 255268, 2005.

(2013). Diretrizes de atenção à reabilitação da pessoa com traumatismo cranioencefálico / Ministério da Saúde. Secretaria de Atenção à Saúde. Departamento de Ações Programáticas Estratégicas. . 148.

24 Sosin, D.M.; Sniezek, J.E.; et al. Trends in death associated with traumatic brain injury, 1979 through 1992. Success and failure. JAMA, v. 273, n. 22, p. 1778-80, 1995.

25 Lu, J.; Marmarou, A.; et al. Mortality from traumatic brain injury. Acta Neurochir Suppl, v. 95, p. 281-5, 2005. 
26 Gerber, L.M.; Chiu, Y.-L.; et al. Marked reduction in mortality in patients with severe traumatic brain injury. J Neurosurg, v. 119, n. 6, p. 1583-90, 2013.

27 McAllister, T.W.; Sparling, M.B.; et al. Differential working memory load effects after mild traumatic brain injury. Neuroimage, v. 14, n. 5, p. 1004-12, 2001.

28 Alves, W.; Macciocchi, S.; et al. Postconcussive symptoms after uncomplicated mild head injury. J Head Trauma Rehabil, v. 8, n. 3, p. 4859, 1993.

29 Guidelines for the management of severe traumatic brain injury. $\mathbf{J}$ Neurotrauma, v. 24, n. suppl 1, p. S1-106, 2007.

30 Reis, H.F.C. dos; Almeida, M.L.O.; et al. Extubation failure influences clinical and functional outcomes in patients with traumatic brain injury. $\mathbf{J}$ Bras Pneumol, v. 39, n. 3, p. 330-8, 2013.

31 Johnstone, B.; Mount, D.; et al. Financial and vocational outcomes 1 year after traumatic brain injury. Arch Phys Med Rehabil, v. 84, n. 2, p. 238-41, 2003.

32 Bednarik, J.; Lukas, Z.; et al. Critical illness polyneuromyopathy: the electrophysiological components of a complex entity. Intensive Care Med, v. 29, n. 9, p. 1505-14, 2003.

33 Stevens, R.D.; Marshall, S.A.; et al. A framework for diagnosing and classifying intensive care unit-acquired weakness. Crit Care Med, v. 37, n. 10 Suppl, p. S299-S308, 2009.

Osler, W. (1892). Principles and practice of medicine.

35 Bolton, C.F.; Gilbert, J.J.; et al. Polyneuropathy in critically ill patients. J Neurol Neurosurg Psychiatry, v. 47, n. 11, p. 1223-31, 1984.

36 Zochodne, D.; Bolton, C.; et al. Myopathy in critical illness. Muscle Nerve, v. 9, p. 652, 1986.

37 Bolton, C.F. Neuromuscular manifestations of critical illness. Muscle Nerve, v. 32, n. 2, p. 140-63, 2005.

38 Latronico, N.; Fenzi, F.; et al. Critical illness myopathy and neuropathy. Lancet, v. 347, n. 9015, p. 1579-82, 1996.

39 Zink, W.; Kollmar, R.; et al. Critical illness polyneuropathy and myopathy in the intensive care unit. Nat Rev Neurol, v. 5, n. 7, p. 372-9, 2009.

40 Kress, J.P.; Hall, J.B. ICU-acquired weakness and recovery from critical illness. N Engl J Med, v. 370, n. 17, p. 1626-35, 2014. 
41 Cartwright, M.S.; Kwayisi, G.; et al. Quantitative neuromuscular ultrasound in the intensive care unit. Muscle Nerve, v. 47, n. 2, p. 255-9, 2013.

42 Witt, N.J.; Zochodne, D.W.; et al. Peripheral nerve function in sepsis and multiple organ failure. Chest, v. 99, n. 1, p. 176-84, 1991.

43 Lacomis, D.; Petrella, J.T.; et al. Causes of neuromuscular weakness in the intensive care unit: a study of ninety-two patients. Muscle Nerve, v. 21, n. 5, p. 610-7, 1998.

$44 \quad$ Nanas, S.; Kritikos, K.; et al. Predisposing factors for critical illness polyneuromyopathy in a multidisciplinary intensive care unit. Acta Neurol Scand, v. 118, n. 3, p. 175-81, 2008.

45 Fenzi, F.; Latronico, N.; et al. Enhanced expression of E-selectin on the vascular endothelium of peripheral nerve in critically ill patients with neuromuscular disorders. Acta Neuropathol, v. 106, n. 1, p. 75-82, 2003.

46 Hermans, G.; Wilmer, A.; et al. Impact of intensive insulin therapy on neuromuscular complications and ventilator dependency in the medical intensive care unit. Am J Respir Crit Care Med, v. 175, n. 5, p. 480-9, 2007.

47 Berghe, G. van den; Wouters, P.; et al. Intensive insulin therapy in critically ill patients. N Engl J Med, v. 345, n. 19, p. 1359-67, 2001.

48 Jonghe, B. De; Sharshar, T.; et al. Paresis acquired in the intensive care unit: a prospective multicenter study. JAMA, v. 288, n. 22, p. 2859-67, 2002.

49 Letter, M.A. de; Schmitz, P.I.; et al. Risk factors for the development of polyneuropathy and myopathy in critically ill patients. Crit Care Med, v. 29, n. 12, p. 2281-6, 2001.

50 Campellone, J. V; Lacomis, D.; et al. Acute myopathy after liver transplantation. Neurology, v. 50, n. 1, p. 46-53, 1998.

51 Patel, B.K.; Pohlman, A.S.; et al. Impact of early mobilization on glycemic control and ICU-acquired weakness in critically ill patients who are mechanically ventilated. Chest, v. 146, n. 3, p. 583-9, 2014.

52 Coakley, J.H.; Nagendran, K.; et al. Preliminary observations on the neuromuscular abnormalities in patients with organ failure and sepsis. Intensive Care Med, v. 19, n. 6, p. 323-8, 1993.

53 Ali, N.A.; O'Brien, J.M.; et al. Acquired weakness, handgrip strength, and mortality in critically ill patients. Am J Respir Crit Care Med, v. 178, n. 3, p. 261-8, 2008.

54 Sharshar, T.; Bastuji-Garin, S.; et al. Presence and severity of intensive 
care unit-acquired paresis at time of awakening are associated with increased intensive care unit and hospital mortality. Crit Care Med, v. 37, n. 12, p. 3047-53, 2009.

55 Tennilä, A.; Salmi, T.; et al. Early signs of critical illness polyneuropathy in ICU patients with systemic inflammatory response syndrome or sepsis. Intensive Care Med, v. 26, n. 9, p. 1360-3, 2000.

56 Hermans, G.; Jonghe, B. De; et al. Clinical review: Critical illness polyneuropathy and myopathy. Crit Care, v. 12, n. 6, p. 238, 2008.

57 Bird, S.J. Diagnosis and management of critical illness polyneuropathy and critical illness myopathy. Curr Treat Options Neurol, v. 9, n. 2, p. 8592, 2007.

58 Latronico, N.; Bertolini, G.; et al. Simplified electrophysiological evaluation of peripheral nerves in critically ill patients: the Italian multi-centre CRIMYNE study. Crit Care, v. 11, n. 1, p. R11, 2007.

59 Khan, J.; Harrison, T.B.; et al. Early development of critical illness myopathy and neuropathy in patients with severe sepsis. Neurology, v. 67, n. 8, p. 1421-5, 2006.

60 Russell, J.A. Management of sepsis. N Engl J Med, v. 355, n. 16, p. 1699-713, 2006.

61 Coakley, J.H.; Nagendran, K.; et al. Patterns of neurophysiological abnormality in prolonged critical illness. Intensive Care Med, v. 24, n. 8, p. 801-7, 1998.

62 Berek, K.; Margreiter, J.; et al. Polyneuropathies in critically ill patients: a prospective evaluation. Intensive Care Med, v. 22, n. 9, p. 849-55, 1996.

63 Hough, C.L.; Steinberg, K.P.; et al. Intensive care unit-acquired neuromyopathy and corticosteroids in survivors of persistent ARDS. Intensive Care Med, v. 35, n. 1, p. 63-8, 2009.

64 Moss, M.; Yang, M.; et al. Screening for critical illness polyneuromyopathy with single nerve conduction studies. Intensive Care Med, v. 40, n. 5, p. 683-90, 2014.

65 Wieske, L.; Verhamme, C.; et al. Feasibility and Diagnostic Accuracy of Early Electrophysiological Recordings for ICU-Acquired Weakness: An Observational Cohort Study. Neurocritical care, 2014.

66 Sander, H.W.; Golden, M.; et al. Quadriplegic areflexic ICU illness: selective thick filament loss and normal nerve histology. Muscle Nerve, $v$. 26, n. 4, p. 499-505, 2002. 
67 Lacomis, D.; Giuliani, M.J.; et al. Acute myopathy of intensive care: clinical, electromyographic, and pathological aspects. Ann Neurol, v. 40, n. 4, p. 645-54, 1996.

68 Faragher, M.W.; Day, B.J.; et al. Critical care myopathy: an electrophysiological and histological study. Muscle Nerve, v. 19, n. 4, p. 516-8, 1996.

69 Hanson, P.; Dive, A.; et al. Acute corticosteroid myopathy in intensive care patients. Muscle Nerve, v. 20, n. 11, p. 1371-80, 1997.

70 Parry, S.M.; El-Ansary, D.; et al. Ultrasonography in the intensive care setting can be used to detect changes in the quality and quantity of muscle and is related to muscle strength and function. J Crit Care, v. 30, n. 5, p. 1151.e9-1151.e14, 2015.

71 Lee, W.-D.; Kim, J.-H.; et al. Differences in Rheobase and Chronaxie between the Paretic and Non-Paretic Sides of Hemiplegic Stroke Patients: a Pilot Study. J Phys Ther Sci, v. 25, n. 6, p. 717-9, 2013.

72 Routsi, C.; Gerovasili, V.; et al. Electrical muscle stimulation prevents critical illness polyneuromyopathy: a randomized parallel intervention trial. Crit Care, v. 14, n. 2, p. R74, 2010.

73 Rodriguez, P.O.; Setten, M.; et al. Muscle weakness in septic patients requiring mechanical ventilation: Protective effect of transcutaneous neuromuscular electrical stimulation. J Crit Care, v. 27, n. 3, p. 1-8, 2011.

74 Zanotti, E.; Felicetti, G.; et al. Peripheral muscle strength training in bedbound patients with COPD receiving mechanical ventilation: effect of electrical stimulation. Chest, v. 124, n. 1, p. 292-6, 2003.

75 Gerovasili, V.; Stefanidis, K.; et al. Electrical muscle stimulation preserves the muscle mass of critically ill patients: a randomized study. Crit Care, v. 13, n. 5, p. R161, 2009.

76 Meesen, R.L.J.; Dendale, P.; et al. Neuromuscular Electrical Stimulation As a Possible Means to Prevent Muscle Tissue Wasting in Artificially Ventilated and Sedated Patients in the Intensive Care Unit: A Pilot Study. Neuromodulation, v. 13, n. 4, p. 315-321, 2010.

77 Dow, D.E.; Cederna, P.S.; et al. Number of contractions to maintain mass and force of a denervated rat muscle. Muscle Nerve, v. 30, n. 1, p. 7786, 2004.

78 Russo, T.L.; Peviani, S.M.; et al. Electrical stimulation based on chronaxie reduces atrogin-1 and myoD gene expressions in denervated rat muscle. Muscle Nerve, v. 35, n. 1, p. 87-97, 2007. 
79 Lapicque, L. Définition expérimentale de l'excitabilité. Comptes Rendus Soc Biol, v. 77, p. 280-3, 1909.

80 Kiernan, M.C.; Burke, D.; et al. Multiple measures of axonal excitability: a new approach in clinical testing. Muscle Nerve, v. 23, n. 3, p. 399-409, 2000.

81 Connolly, B.A.; Jones, G.D.; et al. Clinical predictive value of manual muscle strength testing during critical illness: an observational cohort study. Crit Care, v. 17, n. 5, p. R229, 2013.

82 Puthucheary, Z.A.; Phadke, R.; et al. Qualitative Ultrasound in Acute Critical Illness Muscle Wasting. Crit Care Med, v. 43, n. 8, p. 1603-11, 2015.

83 Grimm, A.; Teschner, U.; et al. Muscle ultrasound for early assessment of critical illness neuromyopathy in severe sepsis. Crit Care, v. 17, n. 5, p. R227, 2013.

84 Tillquist, M.; Kutsogiannis, D.J.; et al. Bedside ultrasound is a practical and reliable measurement tool for assessing quadriceps muscle layer thickness. JPEN J Parenter Enteral Nutr, v. 38, n. 7, p. 886-90, 2014.

85 Baldwin, C.E.; Paratz, J.D.; et al. Diaphragm and peripheral muscle thickness on ultrasound: intra-rater reliability and variability of a methodology using non-standard recumbent positions. Respirology, v. 16, n. 7, p. 113643, 2011.

86 Seymour, J.M.; Ward, K.; et al. Ultrasound measurement of rectus femoris cross-sectional area and the relationship with quadriceps strength in COPD. Thorax, v. 64, n. 5, p. 418-23, 2009.

87 Gruther, W.; Benesch, T.; et al. Muscle wasting in intensive care patients: ultrasound observation of the $\mathbf{M}$. quadriceps femoris muscle layer. $\mathbf{J}$ Rehabil Med, v. 40, n. 3, p. 185-9, 2008.

88 Manning, E.M.; Shenkin, A. Nutritional assessment in the critically ill. Crit Care Clin, v. 11, n. 3, p. 603-34, 1995.

89 Walton, J.M.; Roberts, N.; et al. Measurement of the quadriceps femoris muscle using magnetic resonance and ultrasound imaging. $\mathbf{B r} \mathbf{J}$ Sports Med, v. 31, n. 1, p. 59-64, 1997.

90 Dupont, A.C.; Sauerbrei, E.E.; et al. Real-time sonography to estimate muscle thickness: comparison with MRI and CT. J Clin Ultrasound, v. 29, n. 4, p. 230-6, 2001.

91 Bunnell, A.; Ney, J.; et al. Quantitative neuromuscular ultrasound in intensive care unit-acquired weakness: A systematic review. Muscle Nerve, 
v. 52, n. 5, p. 701-8, 2015.

92 Sarwal, A.; Parry, S.M.; et al. Interobserver Reliability of Quantitative Muscle Sonographic Analysis in the Critically III Population. J Ultrasound Med, v. 34, n. 7, p. 1191-200, 2015.

93 Chi-Fishman, G.; Hicks, J.E.; et al. Ultrasound imaging distinguishes between normal and weak muscle. Arch Phys Med Rehabil, v. 85, n. 6, p. 980-6, 2004.

94 Freilich, R.J.; Kirsner, R.L.; et al. Isometric strength and thickness relationships in human quadriceps muscle. Neuromuscul Disord, v. 5, n. 5 , p. 415-22, 1995.

95 Botter, A.; Oprandi, G.; et al. Atlas of the muscle motor points for the lower limb: implications for electrical stimulation procedures and electrode positioning. Eur J Appl Physiol, v. 111, n. 10, p. 2461-71, 2011.

96 Park, B.K.; Shin, Y.B.; et al. Anatomic motor point localization of the biceps brachii and brachialis muscles. J Korean Med Sci, v. 22, n. 3, p. 45962, 2007.

97 Arts, I.M.P.; Pillen, S.; et al. Normal values for quantitative muscle ultrasonography in adults. Muscle Nerve, v. 41, n. 1, p. 32-41, 2010.

98 Abramoff, M.; Magalhaes, P.; et al. Image processing with ImageJ. Biophoton Int, v. 11, n. 7, p. 36-42, 2004.

99 Pillen, S.; Tak, R.O.; et al. Skeletal muscle ultrasound: correlation between fibrous tissue and echo intensity. Ultrasound Med Biol, v. 35, n. 3, p. 443-6, 2009.

100 Pillen, S.; Keimpema, M. van; et al. Skeletal muscle ultrasonography: Visual versus quantitative evaluation. Ultrasound Med Biol, v. 32, n. 9, p. 1315-21, 2006.

101 Latronico, N.; Nattino, G.; et al. Validation of the peroneal nerve test to diagnose critical illness polyneuropathy and myopathy in the intensive care unit: the multicentre Italian CRIMYNE-2 diagnostic accuracy study. F1000Research, v. 3, 2014.

102 Brown, A. Axonal transport of membranous and nonmembranous cargoes: a unified perspective. J Cell Biol, v. 160, n. 6, p. 817-21, 2003.

103 Bolton, C.F. Neuromuscular conditions in the intensive care unit. Intensive Care Med, v. 22, n. 9, p. 841-3, 1996.

104 Batt, J.; Santos, C.C. Dos; et al. Muscle injury during critical illness. JAMA, v. 310, n. 15, p. 1569-70, 2013. 
105 Benecke, R.; Berthold, A.; et al. Denervation activity in the EMG of patients with upper motor neuron lesions: time course, local distribution and pathogenetic aspects. J Neurol, v. 230, n. 3, p. 143-51, 1983.

106 Dattola, R.; Girlanda, P.; et al. Muscle rearrangement in patients with hemiparesis after stroke: an electrophysiological and morphological study. Eur Neurol, v. 33, n. 2, p. 109-14, 1993.

107 Segers, J.; Hermans, G.; et al. Feasibility of neuromuscular electrical stimulation in critically ill patients. J Crit Care, v. 29, n. 6, p. 1082-8, 2014.

108 Kho, M.E.; Truong, A.D.; et al. Neuromuscular electrical stimulation in mechanically ventilated patients: a randomized, sham-controlled pilot trial with blinded outcome assessment. J Crit Care, v. 30, n. 1, p. 32-9, 2015.

109 Harper, N.J.; Greer, R.; et al. Neuromuscular monitoring in intensive care patients: milliamperage requirements for supramaximal stimulation. $\mathbf{B r} \mathbf{J}$ Anaesth, v. 87, n. 4, p. 625-7, 2001.

110 Kopman, A.F.; Lawson, D. Milliamperage requirements for supramaximal stimulation of the ulnar nerve with surface electrodes. Anesthesiology, v. 61, n. 1, p. 83-5, 1984.

111 Puthucheary, Z.A.; Rawal, J.; et al. Acute skeletal muscle wasting in critical illness. JAMA, v. 310, n. 15, p. 1591-600, 2013.

112 Caresio, C.; Molinari, F.; et al. Muscle echo intensity: reliability and conditioning factors. Clin Physiol Funct Imaging, v. 35, n. 5, p. 393-403, 2015. 


\section{ANEXO I - Aprovação do Comitê de Ética em Pesquisa com Seres Humanos}

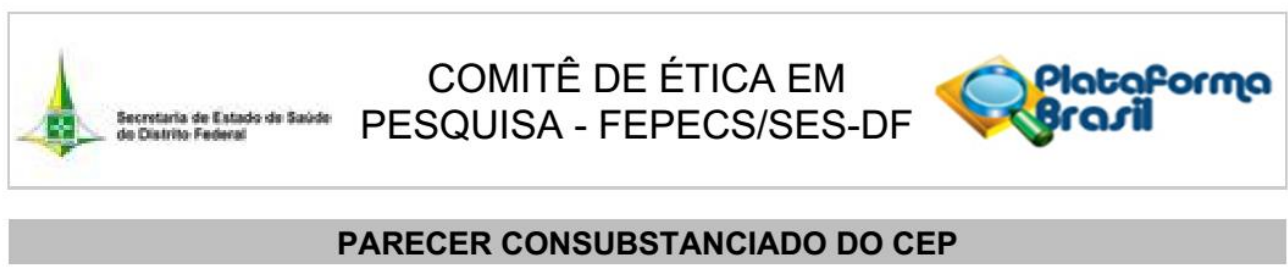

\section{DADOS DO PROJETO DE PESQUISA}

Título da Pesquisa: Estimulação elétrica neuromuscular em pacientes com Traumatismo Crânio Encefálico sob ventilação mecânica prolongada: Ensaio Clínico Randomizado

Pesquisador: Vinicius Zacarias Maldaner da Silva

Área Temática:

Versão: 3

CAAE: 19036013.8 .0000 .5553

Instituição Proponente: DISTRITO FEDERAL SECRETARIA DE SAUDE

Patrocinador Principal: Financiamento Próprio

\section{DADOS DO PARECER}

Número do Parecer: 417.180

Data da Relatoria: 16/09/2013

Considerações sobre os Termos de apresentação obrigatória:

Folha de Rosto: apresentada.

Termo de Concordância: apresentado.

TCLE: apresentado.

Currículo dos pesquisadores: apresentados

Recomendações:

Conclusões ou Pendências e Lista de Inadequações:

Pendências atendidas.

Situação do Parecer:

Aprovado

Necessita Apreciação da CONEP:

Não

Considerações Finais a critério do CEP: 